PHYSICAL REVIEW D 94, 094001 (2016)

\title{
Two-solar-mass hybrid stars: A two model description using the Nambu-Jona-Lasinio quark model
}

\author{
Renan Câmara Pereira, ${ }^{*}$ Pedro Costa, ${ }^{\dagger}$ and Constança Providência \\ CFisUC, Department of Physics, \\ University of Coimbra, P-3004-516 Coimbra, Portugal
}

(Received 6 July 2016; published 2 November 2016)

\begin{abstract}
Hybrid stars with a quark phase described by the Nambu-Jona-Lasinio model are studied. The hadronquark model used to determine the stellar matter equation of state favors the appearance of quark matter: the coincidence of the deconfinement and chiral transitions and a low vacuum constituent quark mass. These two properties are essential to build equations of state that predict pure quark matter in the center of neutron stars. The effect of vector-isoscalar and vector-isovector terms is discussed, and it is shown that the vectorisoscalar terms are necessary to describe $2 M_{\odot}$ hybrid stars, and the vector-isovector terms result in larger quark cores and a smaller deconfinement density.
\end{abstract}

DOI: 10.1103/PhysRevD.94.094001

\section{INTRODUCTION}

Compact stars are natural laboratories to investigate the properties of strongly interacting matter at high densities and small temperatures. Due to their very large central densities, several times larger than normal saturation density, it is possible that the deconfinement phase transition and the partial restoration of chiral symmetry may occur inside compact stars. Indeed, as density increases baryons start to overlap, the distance between quarks becomes very short, and distinct baryons gradually cease to exist. Consequently, inside a compact star the density could be high enough to involve quark degrees of freedom. The study of the behavior of the matter under extreme conditions such as the ones existing in the interior of neutron stars, should take into account that at low densities the relevant degrees of freedom are hadrons while at high densities quark degrees of freedom may set in giving rise to hybrid stars. However, the two solar mass pulsars PSR J0348 $+0432\left(M=2.01 \pm 0.04 M_{\odot}\right)$ [1] and PSR J1614 - 2230 (with the recently updated mass $1.928 \pm 0.017 M_{\odot}[2,3]$ ) set a strong constraint on the high density equation of state $(\mathrm{EoS})$, in particular, on the possible existence of hyperons, kaon condensation or even quark matter inside neutron stars.

In Ref. [4] hybrid stars are described using a two model approach, hadronic matter within a nuclear field theory model and quark matter within the MIT bag model [5]. The hadronquark phase transition is obtained imposing Gibbs conditions and considering global electric charge neutrality, which develops a mixed phase separating a pure hadronic and a pure quark phase. The MIT bag model is a quite simple model that has been widely used. A quark core is possible if the bag constant is not too high. This parameter is constrained from

\footnotetext{
*renan.pereira@student.fisica.uc.pt pcosta@teor.fis.uc.pt *cp@teor.fis.uc.pt
}

below, imposing that at saturation density nuclear matter has a lower energy than strange matter, see Ref. [6].

In Ref. [7], the authors consider, instead of the MIT bag model, the SU(3) Nambu-Jona-Lasinio (NJL) model to describe the quark phase. It is shown that a pure quark phase does not occur inside a neutron star, although quarks might exist as part of a non-homogenous quark-hadronic mixed phase in the center of the star, in stars with a mass close to the maximum allowed mass, $\sim 1.7 M_{\odot}$. As in [4], hadronic matter is described within a relativistic mean field (RMF) model. Similar results are obtained applying a Brueckner Hartree-Fock approach to describe the hadronic phase, and even if a superconducting quark phase is considered for the quark phase within the NJL model [8]. Maximum mass stars obtained have a mass $\sim 1.8 M_{\odot}$, becoming unstable as soon as quark matter sets in. At finite temperature [9] it was possible to obtain pure quark matter in the star center describing quark matter within NJL model but masses below $1.9 M_{\odot}$ were obtained.

However, contrary to [8], a stable cold hybrid star with a diquark condensation in the quark phase was obtained in [10] within a SU(2) NJL model. This different behavior was attributed in [11], to the different vacuum constituent quark masses obtained in both calculations, and, in particular, it was shown that the hadron-quark phase transition is controlled by the constituent mass of the nonstrange quarks in vacuum, and that smaller vacuum constituent masses favor the appearance of a pure quark phase because the zero pressure is shifted to smaller chemical potentials.

A stable cold quark phase has also been obtained within SU(3) NJL model by the introduction of a bag constant, $B^{*}$, which guarantees that the partial restoration of chiral symmetry coincides with the transition from hadronic to the quark matter [12]. This constant shifts the effective bag constant as defined in [13] to smaller values and favors the hadron-quark transition. However, no two solar mass hybrid stars were predicted. In Ref. [14] the fixing condition of the 
bag constant $B^{*}$ was relaxed and the deconfinement baryonic density, which was chosen beforehand, was used to determine the bag constant. Stars with over two solar masses and a quark core in a color super-conducting phase were obtained with a vector interaction added to the NJL Lagrangian density. In Ref. [15] the consequences of quark nucleation were studied and it was shown that not all two solar mass hybrid star configurations are populated after nucleation.

Multiple other studies of the quark-hadron phase transition in neutron stars, involving several approaches for the description of the quark matter and the hadronic matter have been performed. The topical issue [16] includes several articles that review different aspects of this problem. Some other approaches used to describe quark matter are the field correlator method [17], perturbative QCD [18], the chromo-dielectric model $[19,20]$, also a new class of twophase EoS for hybrid stars was discussed in [21]. In [22], a unified approach to the EoS of a hybrid star was proposed with both nuclear and quark matter described within the framework of the NJL model, and, moreover, the internal quark structure of the free nucleon was taken into account. However, stable hybrid stars were only possible with a quite strong pairing interaction and maximum masses below $1.5 M_{\odot}$ were obtained. More recently in [23], hybrid stars were also described in the framework of the NJL model for both the hadronic and the quark phases, but structureless nucleons were considered in the hadronic phase and the couplings were fitted independently in each one of the phases, contrary to [22]. However, in [23], the nucleonic EoS satisfies experimental and theoretical constraints at subsaturation, saturation and suprasaturation densities and $2 M_{\odot}$ stable hybrid stars have been obtained.

The role of the vector interaction, responsible for the excitations of vector and pseudovector mesons, in the properties of compact stars has been extensively studied within the SU(3) NJL model (see for example [12,14,15,23-31]). It is known that for a positive $G_{V}$ the vector interaction provides a repulsive interaction between quarks. This aspect is very important because it stiffens the NJL EoS, which is essential to describe high-mass hybrid stars. Models with a larger $G_{V}$ give larger maximum star masses $[14,26]$.

Concerning the effect of the vector interaction on the QCD phase diagram, namely on the chiral first-order transition, it has been shown that when $G_{V}$ is positive (negative) it contributes to weaken (strengthen) the first-order transition due to repulsive (attractive) nature of the interaction [32]. Indeed, a repulsive interaction shrinks the first-order transition region, which forces the critical endpoint to occur at smaller temperatures, and as $G_{V}$ increases the first-order transition occurs at higher baryonic chemical potentials.

However, in spite of its importance, the value of the vector coupling, $G_{V}$, has not yet been definitively settled: its value in the vacuum can be determined by fitting the vector meson spectrum $[33,34]$ but it is not evident that the value of $G_{V}$ in the medium has to be the same as in the vacuum [32]. In fact, finite-density environment might give rise to a vector interaction, described by a finite $G_{V}$, even though the contribution of this interaction is zero in the vacuum [32]. On the other hand, recent studies of the QCD phase diagram using the extended version of the NJL model with Polyakov loop suggest that the magnitude of $G_{V}$ may be comparable to or larger than the coupling $G_{S}[35,36]$, so as most works we will also consider $G_{V}$ as a free parameter and vary its magnitude in the range $0 \leq G_{V} / G_{S} \leq 1$.

The main objective of the present work is to study the possibility of obtaining two solar mass hybrid stars with a quark core described within the NJL model considering a more generalized interaction than the one used in previous works. The hadronic sector will be described within an RMF model. We will perform a complete study considering both the SU(2) and SU(3) NJL versions, however, only the latter allows the inclusion of strangeness which will probably exist inside compact stars. In fact, it is expected that in the interior of a neutron star strangeness will be present either in the form of hyperons, kaon condensation or deconfined quark matter [4].

Previous studies have shown that a quark phase is favored if a smaller vacuum constituent quark mass than the one obtained with the SU(3) NJL parametrization given in [37] is used and if a bag constant $B^{*}$ is included. We will, therefore, investigate how the choice of the hadron and quark EoS, obtained from two independent models, one for the hadronic phase and another for the quark phase, for the calculation of a hybrid star EoS, allows the description of $2 M_{\odot}$ stars. In particular, we will consider: (a) a low vacuum constitutent quark mass; (b) that the deconfinement phase transition coincides with the partial restoration of chiral symmetry. The first condition is implemented by fitting the NJL parameters including a constraint on the vacuum constituent mass and the second by introducing an effective bag constant, $B^{*}$, which guarantees that the chiral symmetry transition. ${ }^{1}$ coincides with the transition from the hadronic to the quark matter [12]. We will analyze the effect of the vector interaction in the properties of compact stars under the conditions described above.

This work is structured as follows. In Sec. II we present the EoS for hadronic matter used at low densities, and the EoS for quark matter obtained within the SU(3) NJL model including vector interaction [the $\mathrm{SU}(2)$ model is also presented for comparison purposes]. We also discuss the conditions of matter in $\beta$-equilibrium and the Gibbs phase equilibrium conditions together with the procedure used to fix the effective bag constant, $B^{*}$, within NJL models. Section III is devoted to present the results for the possible existence of hybrid stars within the SU(2) NJL model and within the extension to the SU(3) NJL model in order to

\footnotetext{
${ }^{1}$ In the present work chiral symmetry transition refers to the transition to the phase where chiral symmetry is partially restored.
} 
take into account strangeness. Finally, Sec. IV is dedicated to concluding remarks.

\section{FORMALISM}

In order to perform our investigation, quark matter is described by the NJL model, in both SU(2) and SU(3) versions, with vector interactions. The SU(3) NJL model will allow us to explore the influence of strangeness in the quark EoS. Indeed, for densities above $\sim 2-3 \rho_{0}$ there is enough energy in the system for strangeness to become relevant. The central densities inside a neutron star are well above this value, therefore, a model that includes strangeness represents a more realistic study of these systems. Comparing both $\mathrm{SU}(2)$ and $\mathrm{SU}(3)$ versions of the NJL model will allow us to infer the role of strangeness in the system.

Hadron matter is described by a RMF nuclear model. To describe the mixed phase we impose local electric charge neutrality and the Gibbs criteria: the pure hadronic phase and the quark phase are connected to each other through mechanical, thermal and chemical equilibrium.

\section{A. Hadronic matter}

The relativistic mean-field model NL3 $\omega \rho[38,39]$ will be used to describe the hadronic (confined) phase of the system in $\beta$-equilibrium. The Lagrangian density of the model reads

$$
\begin{aligned}
\mathcal{L}= & \sum_{N=p, n} \bar{\psi}_{N}\left[\gamma^{\mu}\left(i \partial_{\mu}-g_{\omega N} \omega_{\mu}-\frac{1}{2} g_{\rho N} \boldsymbol{\tau} \cdot \boldsymbol{\rho}_{\mu}\right)\right. \\
& \left.-\left(m_{N}-g_{\sigma N} \sigma\right)\right] \psi_{N}+\frac{1}{2} \partial^{\mu} \sigma \partial_{\mu} \sigma-\frac{1}{2} m_{\sigma}^{2} \sigma^{2}-\frac{1}{4} \Omega_{\mu \nu} \Omega^{\mu \nu} \\
& +\frac{1}{2} m_{\omega}^{2} \omega^{\mu} \omega_{\mu}-\frac{1}{4} \boldsymbol{\rho}^{\mu \nu} \cdot \boldsymbol{\rho}_{\mu \nu}+\frac{1}{2} m_{\rho}^{2} \boldsymbol{\rho}^{\mu} \cdot \boldsymbol{\rho}_{\mu} \\
& -\frac{1}{3} b m_{N}\left(g_{\sigma N} \sigma\right)^{3}-\frac{1}{4} c\left(g_{\sigma N} \sigma\right)^{4} \\
& +\Lambda_{\omega}\left(g_{\omega}^{2} \omega_{\mu} \omega^{\mu}\right)\left(g_{\rho}^{2} \boldsymbol{\rho}_{\mu} \cdot \boldsymbol{\rho}^{\mu}\right) .
\end{aligned}
$$

This model contains several nonlinear terms: besides the usual cubic and quartic terms on the $\sigma$-meson, there is also a quartic term that mixes the $\omega$ and the $\rho$-meson and which results in a softening of the symmetry energy at large densities. However, since it does not include a quartic term on the $\omega$-meson it has a quite stiff EoS at large densities. No hyperons are included in the present study. The onset of hyperons will certainly compete with the quark onset. But, as shown in Ref. [39] the onset of hyperons for NL3 $\omega \rho$ occurs at $0.31 \mathrm{fm}^{-3}$, above the onset of quark matter as we will see in Sec. III B. Therefore, we will only consider nucleonic matter in the hadronic phase because the appearance of hyperons in some cases only would make the comparisons difficult.

The NL3 $\omega \rho$ model has the following saturation properties (see [38,39]): saturation density $\rho_{0}=0.148 \mathrm{fm}^{-3}$, binding energy $E / A=-16.30 \mathrm{MeV}$, incompressibility $K=271.76 \mathrm{MeV}$, symmetry energy $J=31.7 \mathrm{MeV}$, symmetry energy slope $L=55.5 \mathrm{MeV}$ and effective mass $M^{*} / M=0.60$. In [39] it was shown that this model satisfies a reasonable amount of constraints: experimental, astrophysical and theoretical from microscopic neutron matter calculations. In particular, the maximum possible neutron star mass is $2.75 M_{\odot}$, well above the $2 M_{\odot}$ constraint imposed by the pulsars J1614-2230 and $\mathrm{J} 0348+043$.

\section{B. The NJL model}

The quark phase of the EoS is described within the SU(3) NJL model including, besides the four quark interaction and the 't Hooft determinant that breaks the $U_{A}(1)$ symmetry, vector and pseudovector terms (both vectorisoscalar and vector-isovector will be considered).

The Lagrangian density is written as,

$$
\begin{aligned}
\mathcal{L}= & \bar{\psi}\left(i \partial+\hat{m}+\gamma^{0} \hat{\mu}\right) \psi+G_{S} \sum_{a=0}^{8}\left[\left(\bar{\psi} \lambda^{a} \psi\right)^{2}+\left(\bar{\psi} i \gamma_{5} \lambda^{a} \psi\right)^{2}\right] \\
& -G_{D}\left[\operatorname{det}\left(\bar{\psi}\left(1+\gamma_{5}\right) \psi\right)+\operatorname{det}\left(\bar{\psi}\left(1-\gamma_{5}\right) \psi\right)\right]-\mathcal{L}_{\text {vec }}
\end{aligned}
$$

with,

$$
\begin{aligned}
\mathcal{L}_{\mathrm{vec}}= & G_{\omega}\left[\left(\bar{\psi} \gamma^{\mu} \lambda^{0} \psi\right)^{2}+\left(\bar{\psi} \gamma^{\mu} \gamma_{5} \lambda^{0} \psi\right)^{2}\right] \\
& +G_{\rho} \sum_{a=1}^{8}\left[\left(\bar{\psi} \gamma^{\mu} \lambda^{a} \psi\right)^{2}+\left(\bar{\psi} \gamma^{\mu} \gamma_{5} \lambda^{a} \psi\right)^{2}\right],
\end{aligned}
$$

where $\lambda^{a}(a=1,2 \ldots 8)$ are the Gell-Mann matrices of the $\mathrm{SU}(3)$ group and $\lambda^{0}=\sqrt{\frac{2}{3}} \mathbb{1}$.

The values of the vector-type couplings in Eq. (3) can be fixed by fitting the meson properties in the vacuum [33], however, we will adopt a different strategy. We start by taking three scenarios for $\mathcal{L}_{\text {vec }}$ :

$$
\mathcal{L}_{\text {vec }}=\left\{\begin{array}{lll}
G_{V} \sum_{a=0}^{8}\left[\left(\bar{\psi} \gamma^{\mu} \lambda^{a} \psi\right)^{2}+\left(\bar{\psi} \gamma^{\mu} \gamma_{5} \lambda^{a} \psi\right)^{2}\right], & \text { with } \quad G_{\omega}=G_{\rho}=G_{V} & \mapsto \operatorname{model~NJL}(\mathrm{V}+\mathrm{P}+\mathrm{VI}+\mathrm{PI}) \\
G_{V}\left[\left(\bar{\psi} \gamma^{\mu} \lambda^{0} \psi\right)^{2}+\left(\bar{\psi} \gamma_{5} \gamma^{\mu} \lambda^{0} \psi\right)^{2}\right], & \text { with } \quad G_{\rho}=0 ; G_{\omega}=G_{V} & \mapsto \operatorname{model} \operatorname{NJL}(\mathrm{V}+\mathrm{P}) \\
G_{V} \sum_{a=1}^{8}\left[\left(\bar{\psi} \gamma^{\mu} \lambda^{a} \psi\right)^{2}+\left(\bar{\psi} \gamma^{\mu} \gamma_{5} \lambda^{a} \psi\right)^{2}\right], & \text { with } \quad G_{\omega}=0 ; G_{\rho}=G_{V} & \mapsto \operatorname{model} \operatorname{NJL}(\mathrm{VI}+\mathrm{PI})
\end{array} .\right.
$$


PEREIRA, COSTA, and PROVIDÊNCIA

We will take the ratio $\xi=G_{V} / G_{S}$ as a free parameter, with $G_{S}$ fixed as usual in the NJL-type models. As pointed out in [32], there is still no constraint on $G_{V}$ at finite density, even if there are attempts in that direction [35]. Having no definitive knowledge not even on its sign, $G_{V}$ can be seen as describing effects induced in dense quark matter and might be related to an in-medium modification [32].

We can also argue that the couplings $G_{S}$ and $G_{D}$ are not well constrained in the medium either, but we follow the usual strategy and fix their values to the vacuum meson properties and take these values for all densities (and/or temperatures). ${ }^{2}$

In model NJL $(\mathrm{V}+\mathrm{P}+\mathrm{VI}+\mathrm{PI})$ we take for the vectortype couplings the particular choice $G_{\omega}=G_{\rho} \equiv G_{V}$ (independently of the value of $G_{V}$ this choice makes the $\omega$ and $\rho$ mesons degenerate in the vacuum [34]).

The thermodynamic potential density (subtracting the zero-point energy contribution $\Omega_{0}$ ) for $G_{\omega} \neq G_{\rho}$ is

$$
\begin{aligned}
\Omega-\Omega_{0}= & 2 G_{S}\left(\sigma_{u}^{2}+\sigma_{d}^{2}+\sigma_{s}^{2}\right)-4 G_{D} \sigma_{u} \sigma_{d} \sigma_{s} \\
& -\frac{2}{3} G_{\omega}\left(\rho_{u}+\rho_{d}+\rho_{s}\right)^{2}-G_{\rho}\left(\rho_{u}-\rho_{d}\right)^{2} \\
& -\frac{1}{3} G_{\rho}\left(\rho_{u}+\rho_{d}-2 \rho_{s}\right)^{2}-2 N_{c} \int \frac{d^{3} p}{(2 \pi)^{3}} \\
& \times \sum_{i=u, d, s}\left[E_{i}+T \ln \left(1+e^{-\left(E_{i}+\tilde{\mu}_{i}\right) / T}\right)\right. \\
& \left.+T \ln \left(1+e^{-\left(E_{i}-\tilde{\mu}_{i}\right) / T}\right)\right],
\end{aligned}
$$

where $\sigma_{i}$ is the $i$-quark flavor condensate, $\rho_{i}$ is the $i$-quark flavor density (both presented in the Appendix) and $E_{i}=\sqrt{p^{2}+M_{i}^{2}}$. The effective chemical potentials for the quarks in the general case are given $b^{3}$ :

$$
\begin{aligned}
\tilde{\mu}_{i} & =\mu_{i}-\frac{4}{3}\left[\left(G_{\omega}+2 G_{\rho}\right) \rho_{i}+\left(G_{\omega}-G_{\rho}\right) \rho_{j}+\left(G_{\omega}-G_{\rho}\right) \rho_{k}\right] \\
i & \neq j \neq k \in\{u, d, s\} .
\end{aligned}
$$

In the mean field approximation, we obtain the following gap equations:

$$
\begin{aligned}
M_{i}-m_{i} & =-4 G_{S} \sigma_{i}+2 G_{D} \sigma_{j} \sigma_{k}, \\
i & \neq j \neq k \in\{u, d, s\} .
\end{aligned}
$$

To understand the role of strangeness in neutron stars we will also adopt a SU(2) NJL model with vector interaction (see for example Ref. [41]). Again, we study three cases for vector interactions. In SU(2) they are obtained from Eq. (3) by substituting the Gell-Mann matrices, $\lambda^{a}$, by the $\mathrm{SU}(2)$

\footnotetext{
${ }^{2}$ For example, the study on how the influence of the density in $G_{D_{3}}$ affects mesons properties were made in Ref. [40].

${ }^{3}$ The full expressions for each case are given in the Appendix.
}

PHYSICAL REVIEW D 94, 094001 (2016)

Pauli matrices $\boldsymbol{\tau}$ matrices that act in flavor space (with $\tau^{0}=\mathbb{1}$ ).

The thermodynamic potential density (subtracting the zero-point energy contribution $\Omega_{0}$ ) is now given by

$$
\begin{aligned}
\Omega-\Omega_{0}= & G_{S}\left(\sigma_{u}+\sigma_{d}\right)^{2}-G_{\omega}\left(\rho_{u}+\rho_{d}\right)^{2}-G_{\rho}\left(\rho_{u}-\rho_{d}\right)^{2} \\
& -2 N_{c} \int \frac{d^{3} p}{(2 \pi)^{3}} \sum_{i=u, d}\left[E_{i}+T \ln \left(1+e^{-\left(E_{i}+\tilde{\mu}_{i}\right) / T}\right)\right. \\
& \left.+T \ln \left(1+e^{-\left(E_{i}-\tilde{\mu}_{i}\right) / T}\right)\right] .
\end{aligned}
$$

The replacement of the Gell-Mann matrices in SU(3) by the Pauli matrices in $\mathrm{SU}(2)$ has a direct effect on the effective chemical potentials. Indeed, for the general case $\left(G_{\rho} \neq G_{\omega}\right)$ they became ${ }^{4}$ :

$$
\tilde{\mu}_{i}=\mu_{i}-2 G_{\omega}\left(\rho_{i}+\rho_{j}\right)-4 t_{i} G_{\rho}\left(\rho_{i}-\rho_{j}\right),
$$

where $t_{i}$ is the isospin projection and takes the value $+1 / 2$ for the $u$-quark. Finally, the gap equations are,

$$
M_{i}-m_{i}=-4 G_{S}\left(\sigma_{i}+\sigma_{j}\right), \quad i \neq j \in\{u, d\} .
$$

In the limit $T \rightarrow 0$ matter inside neutron stars is degenerate. The pressure, and the energy density,

$$
\epsilon=\Omega+\sum_{i=u, d} \mu_{i} \rho_{i}
$$

are given in the Appendix.

\section{Parameters of the models}

In the SU(2) NJL model, when equal current masses for each quark flavor are considered, there are three free parameters: the current quark mass $m_{u}=m_{d}=m$, the coupling $G_{S}$, and the cutoff, $\Lambda$, that regularizes the model. Indeed, the NJL model is not renormalizable and there are different ways to regularize the model (see for example [42]). In this work, we will consider a sharp cutoff, $\Lambda$, in 3-momentum space.

The parameters of the model are fixed in order to reproduce the experimental values for the mass and decay constant of the pion $\left(m_{\pi}=135.0 \mathrm{MeV}\right.$ and $f_{\pi}=92.4 \mathrm{MeV}$ ) and the value of the quark condensate in the vacuum.

Since we are interested in studying hybrid neutron stars containing a hadronic and a quark phase, a NJL model parametrization that reproduces in the vacuum the same baryonic chemical potential as the hadronic model should be considered, i.e. a parametrization that gives, in the vacuum, $M_{u}=M_{d} \approx 313 \mathrm{MeV}$, about one third of the vacuum

\footnotetext{
${ }^{4}$ The full expressions for each case in $\mathrm{SU}(2)$ are given in the Appendix.
} 
TABLE I. Sets of parameters used throughout the work and reproduced observables in the vacuum, for each parametrization. $\Lambda$ is the model cutoff, $m_{u, d}$ is the quark current mass, and $G_{S}$ is the coupling constant. The results for the $u$-quark condensate, $\langle\bar{u} u\rangle$, and for the constituent masses, $M_{u, d}$, are also presented.

\begin{tabular}{lccccc}
\hline \hline $\begin{array}{c}\text { Set } \\
\text { SU(2) }\end{array}$ & $\begin{array}{c}\Lambda \\
{[\mathrm{MeV}]}\end{array}$ & $\begin{array}{c}m_{u, d} \\
{[\mathrm{MeV}]}\end{array}$ & $G_{S} \Lambda^{2}$ & $\begin{array}{c}-\langle\bar{u} u\rangle^{1 / 3} \\
{[\mathrm{MeV}]}\end{array}$ & $\begin{array}{c}M_{u, d} \\
{[\mathrm{MeV}]}\end{array}$ \\
\hline \hline
\end{tabular}

nucleon mass. We propose the new set of parameters for the $\mathrm{SU}(2)$ model, see Table I, that gives $m_{\pi}=135 \mathrm{MeV}, f_{\pi}=$ 92.4 $\mathrm{MeV}$ and $\langle\bar{u} u\rangle=(-248.2 \mathrm{MeV})^{3}$.

As already mentioned, the parameter $G_{V}$ in the vectorial terms is seen as a "free" parameter and consequently, in the present work we study several values of the ratio $\xi=G_{V} / G_{S}$.

In the $T=0$ limit for stellar matter application, we define the ratio between the Fermi's moment for each flavor of quark $\left(\lambda_{F_{i}}\right)$, and the model's cutoff $(\Lambda)$ as the limit of applicability of our model: the model is valid for densities and/or chemical potentials that verify $\lambda_{F_{i}} / \Lambda \leq 1$. In SU(2), the studied models are still valid at about $\rho_{B} \approx 11 \rho_{0}$ (where $\rho_{0}=0.16 \mathrm{fm}^{-3}$ is the saturation density), a far larger density than the ones found inside neutron stars.

As previously in the $\mathrm{SU}(2)$ case, we propose a new parametrization for the $\mathrm{SU}(3)$ case which reproduces the same baryonic chemical potential at zero density in both quark and hadronic phases (implying that $M_{u}=M_{d} \approx 313 \mathrm{MeV}$ ). This new parametrization is presented in Table II. In Table III, we compare the values of the calculated observables with the respective experimental values.

As in the $\mathrm{SU}(2)$ case, we restrict the applicability of the models in SU(3), in the $T \rightarrow 0$ limit, to the density at which the ratio $\lambda_{F_{i}} / \Lambda \leq 1$. The models in $\mathrm{SU}(3)$ are valid until at least $15 \rho_{0}$, densities well above those found inside neutron stars.

\section{C. $\beta$-equilibrium matter}

In order to study cold stellar matter, $\beta$-equilibrium and charge neutral matter must be imposed and, therefore, a leptonic contribution must be added to the Lagrangian densities (1) and (2),

TABLE II. $\Lambda$ is the model cutoff, $m_{u, d}$ and $m_{s}$ are the quark current masses, $G_{S}$ and $G_{D}$ are coupling constants. $M_{u, d}$ and $M_{s}$ are the resulting constituent quark masses in the vacuum.

\begin{tabular}{|c|c|c|c|c|c|c|c|}
\hline Set & $\begin{array}{c}\Lambda \\
{[\mathrm{MeV}]}\end{array}$ & $\begin{array}{c}m_{u, d} \\
{[\mathrm{MeV}]}\end{array}$ & $\begin{array}{c}m_{s} \\
{[\mathrm{MeV}]}\end{array}$ & $G_{S} \Lambda^{2}$ & $G_{D} \Lambda^{5}$ & $\begin{array}{c}M_{u, d} \\
{[\mathrm{MeV}]}\end{array}$ & $\begin{array}{c}M_{s} \\
{[\mathrm{MeV}]}\end{array}$ \\
\hline SU(3) & 630.0 & 5.5 & 135.7 & 1.781 & 9.29 & 312.2 & 508 \\
\hline
\end{tabular}

TABLE III. Masses and decay constants of several mesons within the model and the respective experimental values.

\begin{tabular}{lrc}
\hline \hline & SU(3) & Experimental [43] \\
\hline$m_{\pi^{ \pm}}[\mathrm{MeV}]$ & 138.5 & 139.6 \\
$f_{\pi^{ \pm}}[\mathrm{MeV}]$ & 90.7 & 92.2 \\
$m_{K^{ \pm}}[\mathrm{MeV}]$ & 493.5 & 493.7 \\
$f_{K^{ \pm}}[\mathrm{MeV}]$ & 96.3 & 110.4 \\
$m_{\eta}[\mathrm{MeV}]$ & 478.2 & 547.9 \\
$m_{\eta^{\prime}}[\mathrm{MeV}]$ & 953.7 & 957.8 \\
\hline \hline
\end{tabular}

$$
\mathcal{L}_{l}=\sum_{l=e, \mu} \bar{\psi}_{l}\left(i \partial+m_{l}\right) \psi_{l}
$$

The leptonic contribution to thermodynamic potential densities of the models considered is

$$
\begin{aligned}
\Omega_{l}= & 2 T \sum_{l=e, \mu} \int \frac{d^{3} p}{(2 \pi)^{3}}\left[\ln \left(1+e^{-\left(E_{l}+\mu_{l}\right) / T}\right)\right. \\
& \left.+\ln \left(1+e^{-\left(E_{l}-\mu_{l}\right) / T}\right)\right],
\end{aligned}
$$

where $E_{l}=\sqrt{p^{2}+m_{l}^{2}}$, and the sum is over electrons and muons. At $T=0$ the mean free path of neutrinos is larger than the star radius and we will consider that they escape and that they have a zero chemical potential.

Neutrality and $\beta$-equilibrium for the hadronic matter results in the conditions

$$
\rho_{p}=\rho_{e}+\rho_{\mu}
$$

and

$$
\mu_{n}-\mu_{p}=\mu_{e},
$$

The corresponding conditions for quark matter read

$$
\frac{1}{3}\left(2 \rho_{u}-\rho_{d}-\rho_{s}\right)-\rho_{e}-\rho_{\mu}=0
$$

and,

$$
\mu_{d}=\mu_{s}=\mu_{u}+\mu_{e}
$$

In the SU(2) NJL model, $s$-quarks are not present and, therefore, $\rho_{s}=0$.

All thermodynamic quantities of interest, e.g. the pressure and the energy density are presented in the Appendix (in the limit $T \rightarrow 0$ ). 


\section{Phenomenological bag constant and Gibbs construction}

As pointed out in Ref. [12] the pressure within the NJLtype models is defined up to a constant $B$, similar to the MIT bag constant. This constant is usually fixed by requiring that the corrected pressure $P$ goes to zero at vanishing baryonic chemical potential (a detailed study of the bag pressure in NJL model was done in Ref. [9]).

However, the procedure used to fix the effective bag constant within NJL models is crucial for the stability of the star when the phase transition to quark matter is considered. In the same work [12], the bag constant $B^{*}$ is introduced and is fixed imposing that the deconfinement occurs at the same baryonic chemical potential, $\mu_{B}^{\text {crit }}$, as the chiral phase transition. In the present work we consider the NL3 $\omega \rho$ model (see Sec. II A) to describe the hadronic phase and compute the transition to quark matter imposing Gibbs conditions and the coincidence between the deconfinement phase transition and the partial restoration of the chiral symmetry. This is achieved by adding to the quark EoS [Eq. (5) in SU(3) and Eq. (8) in SU(2)] the suitable value of the bag constant, $B^{*}$. For comparison we will also study the $B^{*}=0$ case. Including $B^{*}$ modifies the quark matter EoS in the following way:

$$
P_{\mathrm{eff}}=P+B^{*}, \quad \epsilon_{\mathrm{eff}}=\epsilon-B^{*}
$$

and, therefore, shifts the pressure to larger values for a given baryonic chemical potential, favoring the hadronquark phase transition.

To build the hybrid EoS we use the Gibbs conditions: both phases must be in chemical, thermal and mechanical equilibrium

$$
\mu_{B}^{H}=\mu_{B}^{Q} \wedge p_{B}^{H}=p_{B}^{Q} \wedge T_{B}^{H}=T_{B}^{Q}=0,
$$

where the $H$ and $Q$ indices represent, respectively, the confined (hadronic) and deconfined (quark) phases.

The chiral symmetry transition point $\left(\mu_{B}^{\text {crit }}\right)$ is defined in the following way: if the phase transition is of first-order, we search for the $\mu_{B}$ at which there is a discontinuity in the quark condensate (the order parameter): the stable solutions of the gap equations are realized by the minimum of the thermodynamic potential or, equivalently, maximum of the pressure (see Ref. [44] for details). If the transition is a crossover, we search for the zeros of the second derivative of the light quark condensates, $\partial^{2}\left\langle\bar{q}_{i} q_{i}\right\rangle / \partial \mu_{B}{ }^{2}=0$. In the cases where there are different chemical potentials for each quark flavor (different phase transitions for each flavor), the chemical potential used in the Gibbs condition is given by the average of the baryonic chemical potentials at the corresponding phase transitions.

$$
\mu_{B}^{\mathrm{crit}}=\frac{\mu_{B(u)}^{\mathrm{crit}}+\mu_{B(d)}^{\mathrm{crit}}}{2} .
$$

\section{RESULTS AND DISCUSSION}

In the present section we present our results and discuss the possible existence of hybrid stars within the NJL model, for the three scenarios previously defined. The neutron star mass and radius are obtained solving the TolmannOppenheimer-Volkov (TOV) equations $[45,46]$. In particular, for each star we calculate the maximum gravitational mass and the respective central density, radius and maximum baryonic mass. We also investigate the role of strangeness in the EoS. For each case we consider $\xi=G_{V} / G_{S}=0,0.25,0.5$, and 0.75 , with $G_{S}$ fixed.

\section{A. Results without strangeness}

We first study the SU(2) NJL case, which means that no strangeness is present in the system. We recall that the parameters of the model have been determined so that in the vacuum the model has the same baryonic chemical potential as the hadronic model.

Table IV shows the order of the chiral symmetry transition for different values of $\xi$, which were taken at $B^{*}=0$, but are independent of the bag constant. It can be seen that for the studied values of $\xi \neq 0$, the chiral transition is a crossover instead of a first-order phase transition. Besides, the transition occurs for smaller chemical potentials for $\xi \neq 0$.

Several $\beta$-equilibrium stellar matter EoS with nonzero $B^{*}$, taking into account the hadron-quark phase transition, are shown in Fig. 1 [panels (a), (b), and (c)], for the different vector contributions. These EoS will be used to determine compact star properties in the following discussion. The maximum mass star configuration determines the maximum central density attained in a star described within a given model. Therefore, in these plots the large colored circles indicate the central density of the maximum

TABLE IV. Type of the chiral symmetry phase transition and respective baryonic chemical potential $\left(\mu_{B}^{\text {crit }}\right)$, for each value of $\xi$, model and parameter set.

\begin{tabular}{lccc}
\hline \hline Model $(\mathrm{SU}(2))$ & $\xi$ & Type & $\mu_{B}^{\text {crit }}[\mathrm{MeV}]$ \\
\hline NJL & 0.00 & 1st-order & 1119 \\
NJL $(\mathrm{V}+\mathrm{P}+\mathrm{VI}+\mathrm{PI})$ & 0.25 & Crossover & 1055 \\
& 0.50 & Crossover & 1099 \\
& 0.75 & Crossover & 1149 \\
$\mathrm{NJL}(\mathrm{V}+\mathrm{P})$ & 0.25 & Crossover & 1051 \\
& 0.50 & Crossover & 1089 \\
& 0.75 & Crossover & 1134 \\
$\mathrm{NJL}(\mathrm{VI}+\mathrm{PI})$ & 0.25 & Crossover & 1022 \\
& 0.50 & Crossover & 1025 \\
& 0.75 & Crossover & 1029 \\
\hline \hline
\end{tabular}



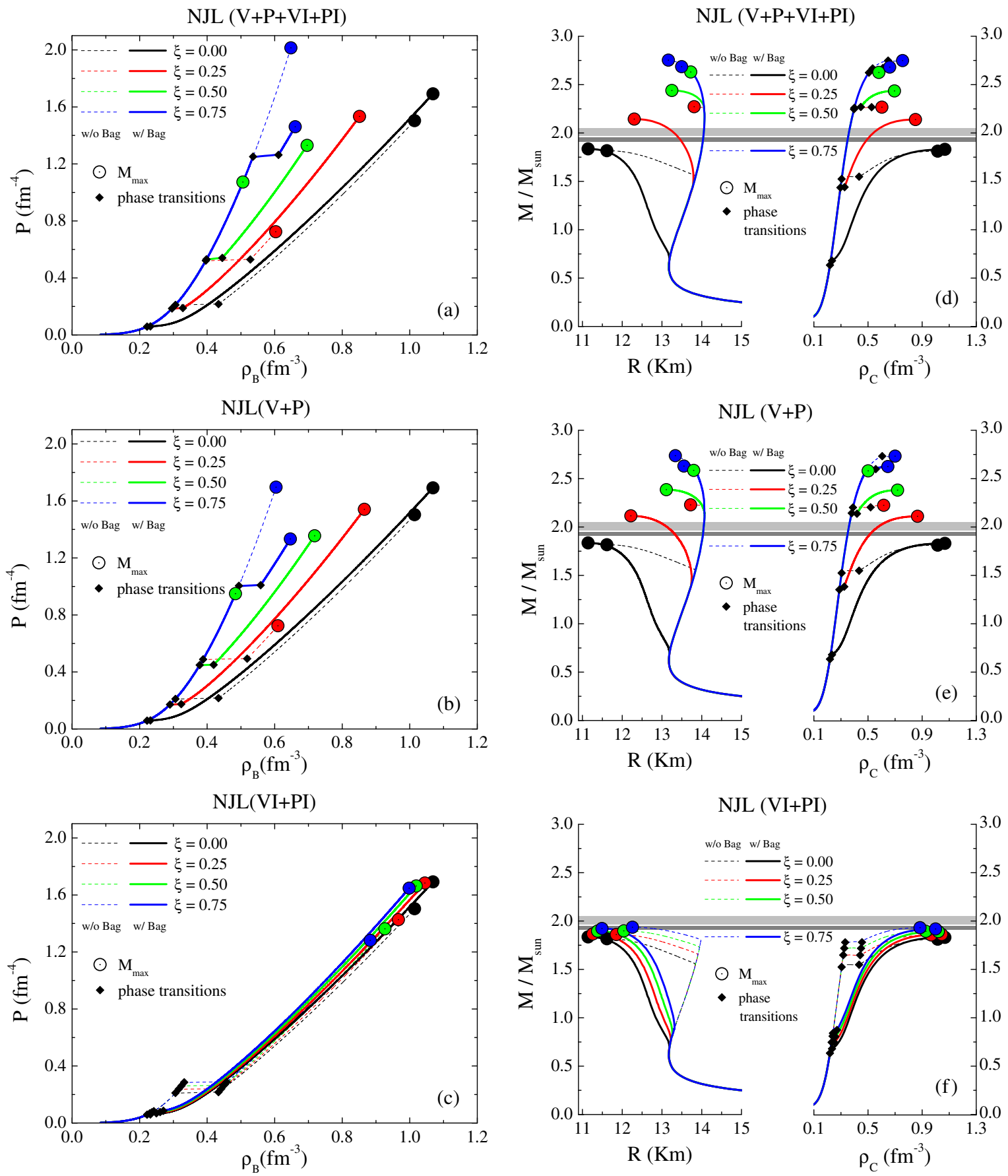

FIG. 1. Left panels: EoS for several values of $\xi$, for the SU(2) models of NJL(V + P + VI + PI) [panel (a)], NJL(V + P) [panel (b)] and NJL(VI + PI) [panel (c)] models. The star maximum mass, central density and confinement-deconfinement phase transitions are highlighted. Right panels: mass-radius and mass-central density diagrams for several values of $\xi$ for the SU(2) NJL(V $+\mathrm{P}+\mathrm{VI}+\mathrm{PI})$ [panel (d)], NJL(V + P) [panel (e)] and NJL(VI + PI) [panel (f)] models. The star maximum mass, central density and confinementdeconfinement phase transitions are highlighted. The light-gray bar represents the mass constraint of the J0348 +043 pulsar $\left(M=2.01 \pm 0.04 M_{\odot}\right)[1]$ while the dark-gray bar the $\mathrm{J} 1614-2230$ pulsar $\left(M=1.928 \pm 0.017 M_{\odot}\right)$ [3]

mass configuration and we do not show the EoS above this density. Small black diamonds indicate the hadron-quark phase transition. In each plot results for both $B^{*}=0$ and $B^{*} \neq 0$ are included. From the analysis of these figures some comments may be drawn: (a) the inclusion of $B^{*} \neq 0$ shifts the deconfinement phase transition to smaller densities, allows the appearance of a quark phase even for a large value of $\xi$ and gives rise to larger central densities; (b) increasing the coupling $\xi$ in models with vector-isoscalar terms makes the EoS harder as shown previously, see $[12,14,24,25]$, and central densities of maximum mass configurations are 
smaller; (c) the vector-isovector term $[\mathrm{NJL}(\mathrm{VI}+\mathrm{PI})]$ has a much smaller effect than the vector-isoscalar term $[\mathrm{NJL}(\mathrm{V}+\mathrm{P})]$, although qualitatively similar; (d) the model labeled $\mathrm{NJL}(\mathrm{P}+\mathrm{V}+\mathrm{PI}+\mathrm{VI})$ incorporates the effects of models $\mathrm{NJL}(\mathrm{P}+\mathrm{V})$ and $\mathrm{NJL}(\mathrm{PI}+\mathrm{VI})$ and, therefore, may give rise to larger central pressures [see panel (a) of Fig. 1]; (e) the harder the quark EoS the larger the deconfinement density, the effect being much stronger if the vector-isoscalar term is included; (f) the EoS which only includes the vector-isovector term originates smaller deconfinement densities and smaller density gaps between the hadronic and the quark density at deconfinement, i.e. a smaller mixed phase. Within this interaction larger central densities, larger quark fractions and smaller radii are attained; $(\mathrm{g})$ for all cases, the vector-isoscalar interaction allows that the star reaches $2 M_{\odot}$ if $\xi$ is large enough (the respective values are given in Table V).

We have calculated the mass and radius of hybrid stars integrating the TOV equations $[45,46]$. In Fig. 1 [panels (d), (e) and (f)], the mass versus radius and mass versus central density curves of the families of stars described by the EoS discussed above are plotted, respectively, in left and right side of each panel. We have considered the Baym-PethickSutherland EoS [47] for the outer crust and for the inner crust the inner crust NL3 $\omega \rho$ EoS that describes the pasta phases within a Thomas-Fermi approach [48] and links smoothly to the core NL3 $\omega \rho$ EoS.

Some properties of the hybrid stars, in particular of the maximum mass configurations are summarized in Table V. These properties include: the bag constant $B^{*}$, the baryonic chemical potential at the transition $\mu_{B}^{H-Q}$, the central baryonic density $\rho^{c}$, the gravitational $M_{m}$ and baryonic mass $M_{b m}$ of the maximum mass configuration, and respective radius $R_{m}$, and the radius of the $1.4 M_{\odot}$ star.

The results show that even taking $B^{*}=0$ we have found stable hybrid stars with a pure quark core at the center $\left(\rho^{c}>\rho^{Q}\right)$. All values of $\xi$ give rise stable hybrid stars if $B^{*} \neq 0$, but for $B^{*}=0$ stable hybrid stars are possible only if the vector-isoscalar interaction is not too strong, (see Table V).

We verify that the vector-isoscalar has a very strong effect on the star structure giving rise to more massive stars, with larger radii and smaller quark contents, while the

TABLE V. Baryonic chemical potential $\left(\mu_{B}^{H-Q}\right)$, hadron $\left(\rho^{H}\right)$ and quark $\left(\rho^{Q}\right)$ baryonic density at deconfinement and respective value of the parameter $B^{*}$. Values of central baryonic density $\left(\rho^{c}\right)$, maximum gravitational mass $\left(M_{m}\right)$, maximum baryonic mass $\left(M_{b m}\right)$, radius $\left(R_{m}\right)$, and radius of the $1.4 M_{\odot}\left(R_{1.4}\right)$, for each model and $\xi$ value, for the different models in SU(2). In bold we present the approximate values of $\xi$ at which $2 M_{\odot}$ are obtained.

\begin{tabular}{|c|c|c|c|c|c|c|c|c|c|c|}
\hline Model & $\xi$ & $\begin{array}{c}B^{*} \\
{\left[\mathrm{MeV} \mathrm{fm}^{-3}\right]}\end{array}$ & $\begin{array}{c}\mu_{B}^{H-Q} \\
{[\mathrm{MeV}]} \\
\end{array}$ & $\begin{array}{c}\rho^{H} \\
{\left[\mathrm{fm}^{-3}\right]}\end{array}$ & $\begin{array}{c}\rho^{Q} \\
{\left[\mathrm{fm}^{-3}\right]}\end{array}$ & $\begin{array}{c}\rho^{c} \\
{\left[\mathrm{fm}^{-3}\right]}\end{array}$ & $\begin{array}{c}M_{m} \\
{\left[\mathrm{M}_{\odot}\right]}\end{array}$ & $\begin{array}{l}M_{b m} \\
{\left[\mathrm{M}_{\odot}\right]}\end{array}$ & $\begin{array}{c}R_{m} \\
{[\mathrm{~km}]}\end{array}$ & $\begin{array}{c}R_{1.4} \\
{[\mathrm{~km}]} \\
\end{array}$ \\
\hline \multirow{2}{*}{$\overline{\mathrm{NJL}}$} & 0.00 & 0 & 1134 & 0.306 & 0.434 & 1.015 & 1.82 & 2.07 & 11.62 & 13.74 \\
\hline & 0.11 & & 1204 & 0.344 & 0.472 & 0.823 & 2.00 & 2.30 & 12.56 & 13.74 \\
\hline \multirow{4}{*}{$\begin{array}{l}\mathrm{NJL} \\
(\mathrm{V}+\mathrm{P}+\mathrm{VI}+\mathrm{PI})\end{array}$} & 0.25 & 0 & 1308 & 0.396 & 0.528 & 0.603 & 2.27 & 2.67 & 13.81 & 13.74 \\
\hline & 0.50 & & 1548 & 0.506 & 0.658 & 0.580 & 2.63 & 3.19 & 13.72 & 13.74 \\
\hline & 0.75 & & 1869 & 0.648 & 0.824 & 0.756 & 2.75 & 3.38 & 13.16 & 13.74 \\
\hline & 0.12 & & 1202 & 0.344 & 0.470 & 0.823 & 2.00 & 2.30 & 12.56 & 13.74 \\
\hline \multirow{4}{*}{$\begin{array}{l}\mathrm{NJL} \\
(\mathrm{V}+\mathrm{P})\end{array}$} & 0.25 & 0 & 1289 & 0.388 & 0.518 & 0.616 & 2.23 & 2.61 & 13.72 & 13.74 \\
\hline & 0.50 & & 1497 & 0.484 & 0.630 & 0.501 & 2.58 & 3.12 & 13.80 & 13.74 \\
\hline & 0.75 & & 1769 & 0.604 & 0.771 & 0.700 & 2.74 & 3.36 & 13.34 & 13.74 \\
\hline & 0.25 & & 1148 & 0.316 & 0.442 & 0.967 & 1.86 & 2.12 & 11.85 & 13.74 \\
\hline \multirow{3}{*}{$\begin{array}{l}\text { NJL } \\
(\mathrm{VI}+\mathrm{PI})\end{array}$} & 0.50 & 0 & 1163 & 0.324 & 0.450 & 0.928 & 1.90 & 2.17 & 12.04 & 13.74 \\
\hline & 0.75 & & 1177 & 0.332 & 0.458 & 0.884 & 1.94 & 2.22 & 12.26 & 13.74 \\
\hline & 1.13 & & 1200 & 0.344 & 0.470 & 0.814 & 2.00 & 2.29 & 12.61 & 13.74 \\
\hline \multirow[t]{2}{*}{ NJL } & 0.00 & 9.84 & 1020 & 0.222 & 0.232 & 1.068 & 1.84 & 2.11 & 11.14 & 12.48 \\
\hline & 0.13 & 12.32 & 1063 & 0.260 & 0.293 & 0.948 & 2.00 & 2.31 & 11.77 & 13.74 \\
\hline \multirow{4}{*}{$\begin{array}{l}\mathrm{NJL} \\
(\mathrm{V}+\mathrm{P}+\mathrm{VI}+\mathrm{PI})\end{array}$} & 0.25 & 15.16 & 1116 & 0.296 & 0.328 & 0.851 & 2.14 & 2.50 & 12.30 & 13.74 \\
\hline & 0.50 & 22.09 & 1313 & 0.398 & 0.445 & 0.695 & 2.44 & 2.91 & 13.25 & 13.74 \\
\hline & 0.75 & 30.84 & 1616 & 0.536 & 0.611 & 0.660 & 2.69 & 3.27 & 13.50 & 13.74 \\
\hline & 0.15 & 12.40 & 1067 & 0.264 & 0.298 & 0.941 & 2.00 & 2.32 & 11.80 & 13.74 \\
\hline \multirow{4}{*}{$\begin{array}{l}\mathrm{NJL} \\
(\mathrm{V}+\mathrm{P})\end{array}$} & 0.25 & 14.50 & 1105 & 0.290 & 0.323 & 0.866 & 2.12 & 2.46 & 12.22 & 13.74 \\
\hline & 0.50 & 20.54 & 1268 & 0.378 & 0.419 & 0.718 & 2.39 & 2.83 & 13.11 & 13.74 \\
\hline & 0.75 & 28.10 & 1519 & 0.494 & 0.558 & 0.647 & 2.63 & 3.19 & 13.55 & 13.74 \\
\hline & 0.25 & 10.29 & 1027 & 0.230 & 0.250 & 1.045 & 1.87 & 2.15 & 11.26 & 12.67 \\
\hline \multirow{3}{*}{$\begin{array}{l}\mathrm{NJL} \\
(\mathrm{VI}+\mathrm{PI})\end{array}$} & 0.50 & 10.75 & 1034 & 0.236 & 0.261 & 1.020 & 1.90 & 2.19 & 11.38 & 12.83 \\
\hline & 0.75 & 11.22 & 1041 & 0.242 & 0.270 & 0.999 & 1.92 & 2.22 & 11.49 & 12.99 \\
\hline & 1.75 & 13.13 & 1074 & 0.268 & 0.301 & 0.921 & 2.00 & 2.33 & 11.92 & 13.74 \\
\hline
\end{tabular}


effect of the vector-isovector term on the maximum mass is very small [as it can be seen in Fig. 1 by comparing panels (d) and (e) with (f)], and to get masses about $\sim 2 M_{\odot}$ high values of $\xi(\sim 1.75)$ are needed, see Table V. However, adding the vector-isoscalar interaction with a weak coupling would be enough to attain $M \gtrsim 2 M_{\odot}$.
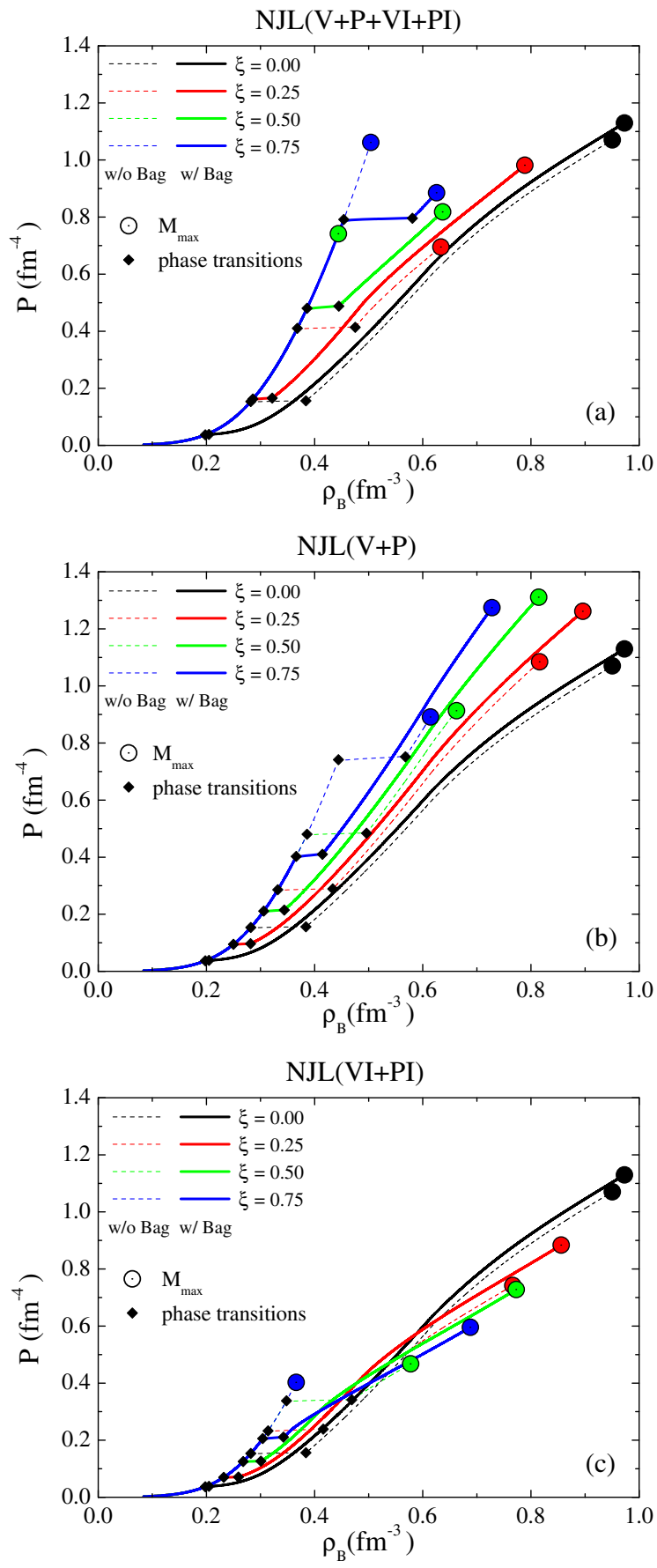

\section{B. The role of strangeness}

In the previous section the strange degree of freedom was not considered, however it is expected that at large densities strangeness will set in. In this section we take strangeness into account, and as before, we will consider a
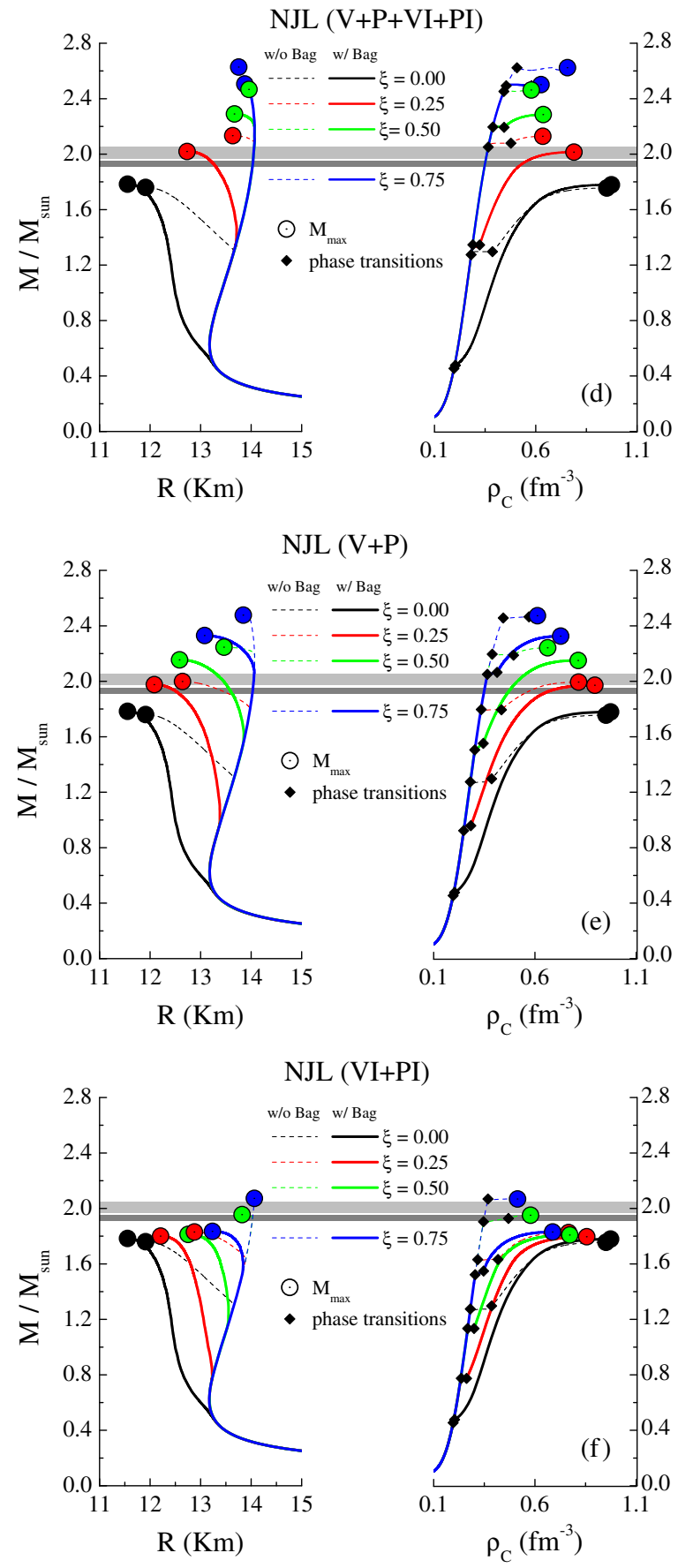

FIG. 2. Left panels: EoS for each value of $\xi$, for the NJL(V + P + VI + PI) [panel (a)], NJL(V + P) [panel (b)] and NJL(VI + PI) [panel (c)] models. The star maximum mass, central density and confinement-deconfinement phase transitions are highlighted. Right panels: mass-radius and mass-central density diagrams for each value of $\xi$ for the $\mathrm{NJL}(\mathrm{V}+\mathrm{P}+\mathrm{VI}+\mathrm{PI})$ [panel (d)], $\mathrm{NJL}(\mathrm{V}+\mathrm{P})$ [panel (e)] and NJL(VI + PI) [panel (f)] models. The star maximum mass, central density and confinement-deconfinement phase transitions are highlighted. The light-gray bar represents the mass constraint of the $\mathrm{J} 0348+043$ pulsar $\left(M=2.01 \pm 0.04 M_{\odot}\right)[1]$ while the dark-gray bar the $\mathrm{J} 1614-2230$ pulsar $\left(M=1.928 \pm 0.017 M_{\odot}\right)$ [3]. 
TABLE VI. Type of the chiral symmetry phase transition and respective baryonic chemical potential $\left(\mu_{B}^{\text {crit }}\right)$, for each value of $\xi$.

\begin{tabular}{lccc}
\hline \hline Model $(\mathrm{SU}(3))$ & $\xi$ & Type & $\mu_{B}^{\text {crit }}[\mathrm{MeV}]$ \\
\hline NJL & 0.00 & 1st-order & 999 \\
NJL $(\mathrm{V}+\mathrm{P}+\mathrm{VI}+\mathrm{PI})$ & 0.25 & Crossover & 1023 \\
& 0.50 & Crossover & 1052 \\
& 0.75 & Crossover & 1087 \\
$\mathrm{NJL}(\mathrm{V}+\mathrm{P})$ & 0.25 & Crossover & 1013 \\
& 0.50 & Crossover & 1028 \\
& 0.75 & Crossover & 1045 \\
NJL(VI + PI $)$ & 0.25 & Crossover & 1008 \\
& 0.50 & Crossover & 1018 \\
& 0.75 & Crossover & 1028 \\
\hline \hline
\end{tabular}

parametrization that predicts a vacuum constituent $u$ and $d$-quark mass equal to $\approx 313 \mathrm{MeV}$, and that describes reasonably well the vacuum properties of several mesons, see Table II. All the features discussed in the previous section remain valid, as we may conclude analysing Table VI where the type of phase transition is given for different strengths of the vector interaction, and Fig. 2 where the EoS [panels (a), (b) and (c)], and the mass/radius and mass/density plots [panels (d), (e) and (f)] are presented. The same conventions of Fig. 1 are adopted.

The effect of $B^{*}$ and $\xi$ are the same as discussed in the previous section within the SU(2) NJL model. It should, however, be referred that care should be taken when comparing the $\mathrm{SU}(2)$ and $\mathrm{SU}(3)$ parametrizations: due to the different normalization of the Pauli and Gell-Mann matrices and the t' Hooft term. Two solar mass stars are obtained if the vector-isoscalar interaction is strong enough, $\xi \gtrsim 0.17-0.28$ depending whether $B^{*}=0$ or $\neq 0$, see Table VII). Including only the vector-isovector interaction, it is not possible to obtain a $2 M_{\odot}$ star with a quark core.

We will next study the onset of strangeness describing quark matter within the SU(3) NJL model. Since the onset of hyperons for NL3 $\omega \rho$ occurs at $0.31 \mathrm{fm}^{-3}$ [39], above the

TABLE VII. Baryonic chemical potential $\left(\mu_{B}^{H-Q}\right)$, hadron $\left(\rho^{H}\right)$ and quark $\left(\rho^{Q}\right)$ baryonic density at deconfinement and respective value of the parameter $B^{*}$. Values of central baryonic density $\left(\rho^{c}\right)$, maximum gravitational mass $\left(M_{m}\right)$, maximum baryonic mass $\left(M_{b m}\right)$, radius $\left(R_{m}\right)$, radius of $1.4 M_{\odot}$ stars $\left(R_{1.4}\right)$, and the ratio of total number of strange quarks to the total baryon number $\left(N_{s} / N_{B}\right)$ [24] of the respective neutron star, for each model and value of $\xi$, for the SU(3) parameter set. In bold we present the approximate values of $\xi$ at which $2 M_{\odot}$ are obtained. The last line corresponds to the combination of $G_{\rho}$ and $G_{\omega}$, in terms of $\xi_{\rho}=G_{\rho} / G_{S}$ and $\xi_{\omega}=G_{\omega} / G_{S}$, at which two solar mass are attained.

\begin{tabular}{|c|c|c|c|c|c|c|c|c|c|c|c|}
\hline Model & $\xi$ & $\begin{array}{c}B^{*} \\
{\left[\mathrm{MeV} \mathrm{fm}^{-3}\right]}\end{array}$ & $\begin{array}{c}\mu_{B}^{H-Q} \\
{[\mathrm{MeV}]} \\
\end{array}$ & $\begin{array}{c}\rho^{H} \\
{\left[\mathrm{fm}^{-3}\right]} \\
\end{array}$ & $\begin{array}{c}\rho^{Q} \\
{\left[\mathrm{fm}^{-3}\right]}\end{array}$ & $\begin{array}{c}\rho^{c} \\
{\left[\mathrm{fm}^{-3}\right]}\end{array}$ & $\begin{array}{c}M_{m} \\
{\left[\mathrm{M}_{\odot}\right]}\end{array}$ & $\begin{array}{l}M_{b m} \\
{\left[\mathrm{M}_{\odot}\right]}\end{array}$ & $\begin{array}{c}R_{m} \\
{[\mathrm{~km}]}\end{array}$ & $\begin{array}{c}R_{1.4} \\
{[\mathrm{~km}]}\end{array}$ & $\begin{array}{c}N_{s} / N_{B} \\
{[\%]} \\
\end{array}$ \\
\hline NJL & $\begin{array}{l}0.00 \\
\mathbf{0 . 1 7}\end{array}$ & 0 & $\begin{array}{l}1093 \\
\mathbf{1 1 9 0}\end{array}$ & $\begin{array}{l}0.282 \\
0.338\end{array}$ & $\begin{array}{l}0.384 \\
0.442\end{array}$ & $\begin{array}{l}0.951 \\
\mathbf{0 . 7 3 4}\end{array}$ & $\begin{array}{l}1.76 \\
\mathbf{2 . 0 0}\end{array}$ & $\begin{array}{l}2.00 \\
\mathbf{2 . 2 9}\end{array}$ & $\begin{array}{l}11.91 \\
\mathbf{1 3 . 0 8}\end{array}$ & $\begin{array}{l}13.39 \\
\mathbf{1 3 . 7 4}\end{array}$ & $\begin{array}{l}1.32 \\
0.93\end{array}$ \\
\hline $\begin{array}{l}\mathrm{NJL} \\
(\mathrm{V}+\mathrm{P}+\mathrm{VI}+\mathrm{PI})\end{array}$ & $\begin{array}{l}0.25 \\
0.50 \\
0.75\end{array}$ & 0 & $\begin{array}{l}1247 \\
1410 \\
1541\end{array}$ & $\begin{array}{l}0.368 \\
0.444 \\
0.504\end{array}$ & $\begin{array}{l}0.475 \\
0.640 \\
0.755\end{array}$ & $\begin{array}{l}0.635 \\
0.578 \\
0.757\end{array}$ & $\begin{array}{l}2.13 \\
2.47 \\
2.63\end{array}$ & $\begin{array}{l}2.48 \\
2.94 \\
3.18\end{array}$ & $\begin{array}{l}13.64 \\
13.96 \\
13.76\end{array}$ & $\begin{array}{l}13.74 \\
13.74 \\
13.74\end{array}$ & $\begin{array}{l}0.53 \\
0.04 \\
0.01\end{array}$ \\
\hline $\begin{array}{l}\text { NJL } \\
(\mathrm{V}+\mathrm{P})\end{array}$ & $\begin{array}{l}\mathbf{0 . 2 5} \\
0.50 \\
0.75\end{array}$ & 0 & $\begin{array}{l}\mathbf{1 1 7 9} \\
1285 \\
1412\end{array}$ & $\begin{array}{l}\mathbf{0 . 3 3 2} \\
0.386 \\
0.444\end{array}$ & $\begin{array}{l}\mathbf{0 . 4 3 4} \\
0.496 \\
0.568\end{array}$ & $\begin{array}{l}\mathbf{0 . 8 1 6} \\
0.663 \\
0.612\end{array}$ & $\begin{array}{l}\mathbf{2 . 0 0} \\
2.25 \\
2.48\end{array}$ & $\begin{array}{l}\mathbf{2 . 3 0} \\
2.63 \\
2.96\end{array}$ & $\begin{array}{l}\mathbf{1 2 . 6 4} \\
13.46 \\
13.85\end{array}$ & $\begin{array}{l}\mathbf{1 3 . 7 4} \\
13.74 \\
13.74\end{array}$ & $\begin{array}{c}\mathbf{0 . 5 0} \\
0.02 \\
\sim 0\end{array}$ \\
\hline $\begin{array}{l}\text { NJL } \\
(\mathrm{VI}+\mathrm{PI})\end{array}$ & $\begin{array}{l}0.25 \\
0.50 \\
\mathbf{0 . 6 0} \\
0.75\end{array}$ & 0 & $\begin{array}{l}1147 \\
1208 \\
\mathbf{1 2 2 5} \\
1243\end{array}$ & $\begin{array}{l}0.314 \\
0.348 \\
\mathbf{0 . 3 5 6} \\
0.366\end{array}$ & $\begin{array}{l}0.416 \\
0.469 \\
\mathbf{0 . 5 0 7} \\
0.558\end{array}$ & $\begin{array}{l}0.766 \\
0.578 \\
\mathbf{0 . 4 2 9} \\
0.515\end{array}$ & $\begin{array}{l}1.83 \\
1.96 \\
\mathbf{2 . 0 0} \\
2.07\end{array}$ & $\begin{array}{l}2.08 \\
2.24 \\
\mathbf{2 . 3 0} \\
2.39\end{array}$ & $\begin{array}{l}12.88 \\
13.82 \\
\mathbf{1 4 . 0 0} \\
14.07\end{array}$ & $\begin{array}{l}13.74 \\
13.74 \\
\mathbf{1 3 . 7 4} \\
13.74\end{array}$ & $\begin{array}{l}1.80 \\
0.85 \\
0.31 \\
0.01\end{array}$ \\
\hline NJL & $\begin{array}{l}0.00 \\
\mathbf{0 . 2 2}\end{array}$ & $\begin{array}{l}6.60 \\
9.49\end{array}$ & $\begin{array}{c}999 \\
\mathbf{1 0 8 7}\end{array}$ & $\begin{array}{l}0.198 \\
0.278\end{array}$ & $\begin{array}{l}0.205 \\
\mathbf{0 . 3 1 5}\end{array}$ & $\begin{array}{l}0.974 \\
\mathbf{0 . 8 0 6}\end{array}$ & $\begin{array}{l}1.78 \\
\mathbf{2 . 0 0}\end{array}$ & $\begin{array}{l}2.05 \\
\mathbf{2 . 2 9}\end{array}$ & $\begin{array}{l}11.55 \\
\mathbf{1 2 . 6 1}\end{array}$ & $\begin{array}{l}12.33 \\
\mathbf{1 3 . 6 3}\end{array}$ & $\begin{array}{l}1.43 \\
2.07\end{array}$ \\
\hline $\begin{array}{l}\mathrm{NJL} \\
(\mathrm{V}+\mathrm{P}+\mathrm{VI}+\mathrm{PI})\end{array}$ & $\begin{array}{l}0.25 \\
0.50 \\
0.75 \\
0.25\end{array}$ & $\begin{array}{r}10.09 \\
14.62 \\
20.57 \\
8.61\end{array}$ & $\begin{array}{l}1100 \\
1287 \\
1431 \\
1049\end{array}$ & $\begin{array}{l}0.286 \\
0.386 \\
0.454 \\
0.250\end{array}$ & $\begin{array}{l}0.322 \\
0.445 \\
0.581 \\
0.282\end{array}$ & $\begin{array}{l}0.789 \\
0.637 \\
0.626 \\
0.896\end{array}$ & $\begin{array}{l}2.02 \\
2.29 \\
2.51 \\
1.98\end{array}$ & $\begin{array}{l}2.33 \\
2.69 \\
3.00 \\
2.28\end{array}$ & $\begin{array}{l}12.73 \\
13.67 \\
13.88 \\
12.08\end{array}$ & $\begin{array}{l}13.71 \\
13.74 \\
13.74 \\
13.26\end{array}$ & $\begin{array}{l}2.14 \\
1.85 \\
0.46 \\
0.98\end{array}$ \\
\hline $\begin{array}{l}\mathrm{NJL} \\
(\mathrm{V}+\mathrm{P})\end{array}$ & $\begin{array}{l}\mathbf{0 . 2 8} \\
0.50 \\
0.75\end{array}$ & $\begin{array}{r}\mathbf{8 . 8 5} \\
10.92 \\
13.63\end{array}$ & $\begin{array}{l}\mathbf{1 0 5 7} \\
1132 \\
1246\end{array}$ & $\begin{array}{l}\mathbf{0 . 2 5 6} \\
0.306 \\
0.366\end{array}$ & $\begin{array}{l}\mathbf{0 . 2 9 0} \\
0.344 \\
0.414\end{array}$ & $\begin{array}{l}\mathbf{0 . 8 8 5} \\
0.814 \\
0.727\end{array}$ & $\begin{array}{l}2.00 \\
2.15 \\
2.33\end{array}$ & $\begin{array}{l}\mathbf{2 . 3 1} \\
2.51 \\
2.75\end{array}$ & $\begin{array}{l}\mathbf{1 2 . 1 4} \\
12.58 \\
13.08\end{array}$ & $\begin{array}{l}\mathbf{1 3 . 3 5} \\
13.74 \\
13.74\end{array}$ & $\begin{array}{l}\mathbf{0 . 9 1} \\
0.48 \\
0.12\end{array}$ \\
\hline $\begin{array}{l}\text { NJL } \\
(\mathrm{VI}+\mathrm{PI})\end{array}$ & $\begin{array}{l}0.25 \\
0.50 \\
0.75\end{array}$ & $\begin{array}{r}7.92 \\
9.33 \\
10.90\end{array}$ & $\begin{array}{l}1029 \\
1072 \\
1129\end{array}$ & $\begin{array}{l}0.232 \\
0.268 \\
0.304\end{array}$ & $\begin{array}{l}0.259 \\
0.301 \\
0.342\end{array}$ & $\begin{array}{l}0.856 \\
0.772 \\
0.688\end{array}$ & $\begin{array}{l}1.80 \\
1.81 \\
1.84\end{array}$ & $\begin{array}{l}2.05 \\
2.06 \\
2.08\end{array}$ & $\begin{array}{l}12.21 \\
12.75 \\
13.24\end{array}$ & $\begin{array}{l}12.95 \\
13.52 \\
13.74\end{array}$ & $\begin{array}{l}2.83 \\
4.12 \\
4.77\end{array}$ \\
\hline$\left\{\begin{array}{l}\xi_{\rho}=0.75 \\
\xi_{\omega}=0.15\end{array}\right.$ & - & 12.44 & 1190 & 0.338 & 0.389 & 0.649 & 2.00 & 2.29 & 13.52 & 13.74 & 3.90 \\
\hline
\end{tabular}


onset of quark matter when $B^{*}$ is included, see Table VII, except for three cases, we will only consider nucleonic matter in the hadronic phase in order to allow a comparison between parametrizations. In Fig. 3 the $s, d$ and $u$ quark fractions $Y_{i}=\rho_{i} /\left(3 \rho_{B}\right)$ are plotted. As soon as the $s$-quark sets in the fraction of $d$-quarks suffers a strong reduction, the fractions of $d$ and $s$-quarks approach $\sim 0.33$, asymptotically, the first from above and the second from below.

Taking the vector-isoscalar interaction alone the strange fraction does not change with $\xi$ [see panel (b) in Fig. 3],
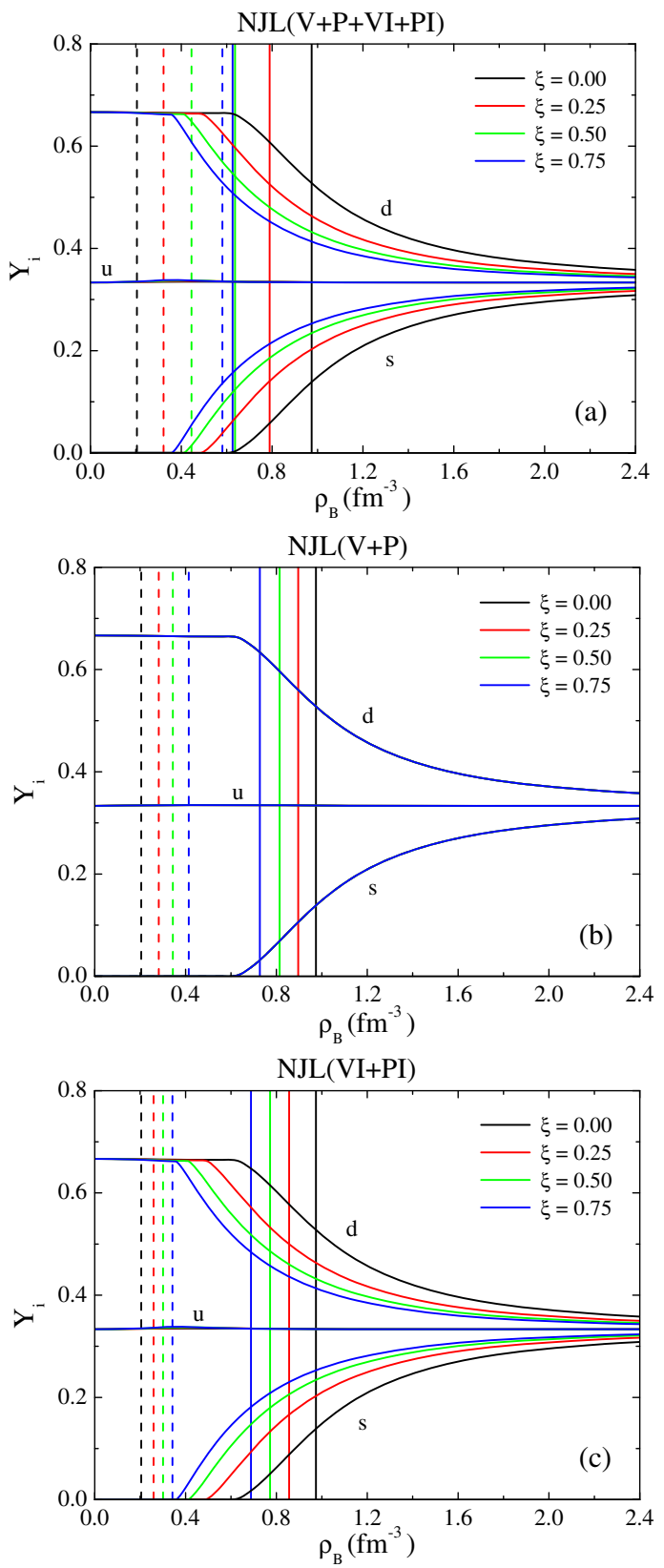

FIG. 3. Fractions of each flavor of quark $\left(Y_{i}\right)$ in function of the baryonic density $\left(\rho_{B}\right)$. The central density $\left(\rho^{c}\right)$ and initial quark phase density $\left(\rho^{Q}\right)$ are shown (full and dashed vertical lines, respectively). The threshold for the emergence of strange quarks in the $\mathrm{NJL}(\mathrm{V}+\mathrm{P})$ model does not depend on $\xi\left(G_{V}\right)$ (black line). which is simply explained because the interaction energy does not depend separately on each flavor [27]. The vectorisovector interaction distinguishes the flavors and the larger $\xi$ the earlier occurs the $s$-quark onset [see panels (a) and (c) of Fig. 3]. The $u$ quark fraction is practically independent of density, with a value close to $1 / 3$, except for a deviation that can be as high as 0.005 if $\xi=0.75$. This deviation from $1 / 3$ is compensated by the presence of electrons in order to turn matter electrically neutral. The onset of strangeness at quite high densities, generally above $3 \rho_{0} \approx 0.5 \mathrm{fm}^{-3}$, is linked to the high constituent mass of the $s$-quark since the partial restoration of chiral symmetry for the $s$-quark occurs at high densities [41].

Properties of hybrid stars, including maximum mass configurations, obtained with the SU(3) parametrization are presented in Table VII with $B^{*}=0$ and $B^{*} \neq 0$. All $B^{*} \neq 0$ cases considered show a pure quark matter in the center of the star. Besides the quantities included in Table V, the fraction of strangeness inside the star is also given. If a large $\xi$ parameter is considered the amount of strangeness in the star is residual except for the NJL(VI + PI) model: in this case the strangeness fraction increases with larger values of $\xi$.

Looking into the details of the NJL(VI + PI) model, we conclude that when $\xi$ is increased the EoS becomes harder before the onset of strangeness: the slope of the curve $P$ versus $\rho$ is larger immediately after the hadron-quark transition [see Fig. 2, panel (c)] allowing stars with a greater mass. However, the higher $\xi$ the lower the density for the onset of strangeness [as already seen in panel (c) of Fig. 3]. After the onset of the $s$-quarks, the EoS becomes softer since the Fermi pressure is distributed among a larger number of degrees of freedom. These two combined effects result in stars with larger masses and lower central densities, but larger fractions of strangeness.

Analyzing the radius of the $1.4 M_{\odot}$ stars obtained within the different parametrizations, see Tables V and VII, we conclude that most of these stars have $R=13.74 \mathrm{~km}$ corresponding to hadronic stars with no quark content. However, some models with $B^{*} \neq 0$ predict the existence of quark matter inside low mass stars with $M<1.4 M_{\odot}$. These stars have the particularity of having smaller radii. In fact, it is possible to get $1.4 M_{\odot}$ stars with $R<13.74 \mathrm{~km}$ within families that predict $2 M_{\odot}$ stars. For SU(3) NJL, the smallest radius obtained is $13.35 \mathrm{~km}$ above the 10.1$11.1 \mathrm{~km}$ prediction of [49] from the analysis of spectroscopic radius measurements during thermonuclear bursts or in quiescence or even the $12.1 \pm 1.1 \mathrm{~km}$ obtained in [50] from experimental constraints and causality restrictions. However, in [51] radii above $13 \mathrm{~km}$ were obtained for $\mathrm{X}$-ray bursting NS and in [52] it has been shown that causality together with the $2 M_{\odot}$ constraints imposes $R>10.7 \mathrm{~km}$. For a recent review of the current status of measurements of radius of neutron stars see [53]. Stronger constraints on neutron star radii are expected from future X-ray telescopes like NICER and Athena. The 

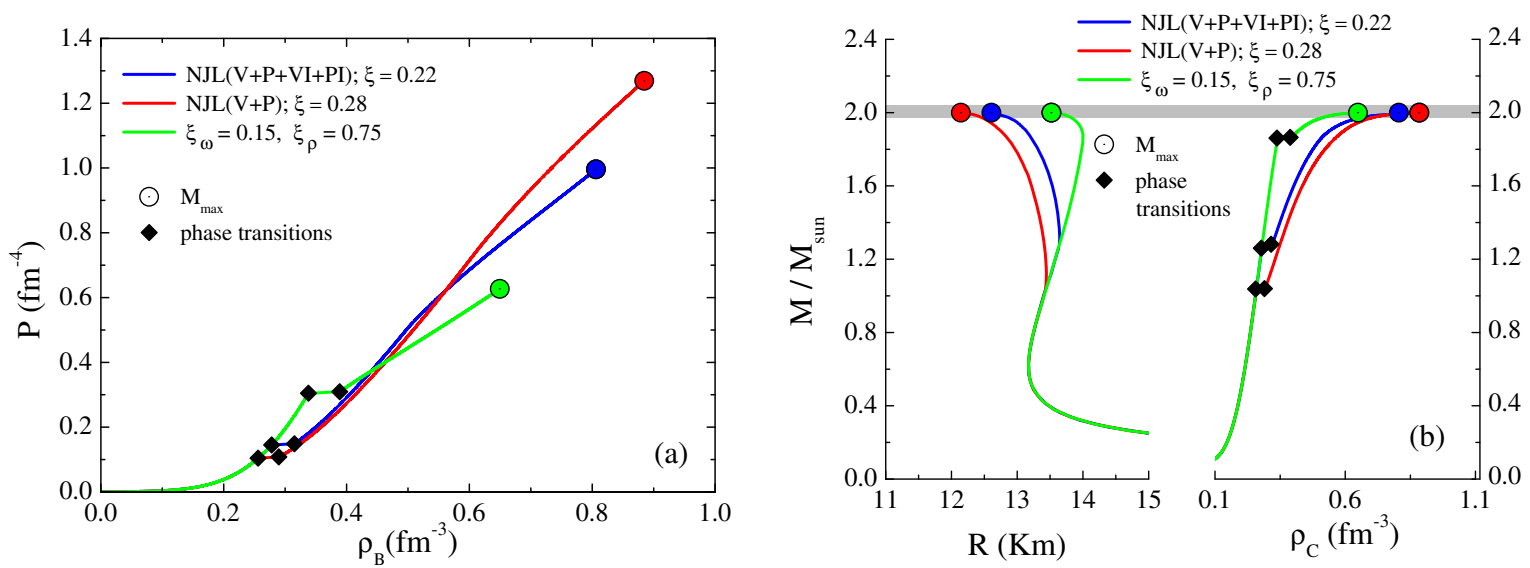

FIG. 4. The EoS (left panel), and the respective mass-radius curves (right panel) of the families of stars having a $2 M_{\odot}$ maximum mass, for three different combinations of $\xi_{\omega}$ and $\xi_{\rho}:\left(\xi_{\omega}, \xi_{\rho}\right)=(0.28,0)$ or NJL $(\mathrm{V}+\mathrm{P})$ with $\xi=0.28,(0.22,0.22)$ or $\mathrm{NJL}(\mathrm{V}+\mathrm{P}+$ $\mathrm{VI}+\mathrm{PI})$ with $\xi=0.22$, and $(0.15,0.75)$.

measurement of the radius of low mass stars such as the pulsar PSR J1918-0642 with a mass the $1.18_{-0.11}^{+0.10} M_{\odot}$ could give some indication on the properties of the EOS at densities just above saturation density and constrain the onset density of quark matter. In the present calculation it is seen that an early onset gives rise to smaller low-mass star radii. However, the radii differences with respect to pure nucleonic matter are probably not strong enough to allow conclusive results mainly because the hadronic EOS itself has still large uncertainties at those densities.

Finally, we present the results for the combination of $G_{\rho}$ and $G_{\omega}$, in terms of $\xi_{\rho}=G_{\rho} / G_{S}$ and $\xi_{\omega}=G_{\omega} / G_{S}$, for which $2.0 M_{\odot}$ are obtained: $\xi_{\rho}=0.75$ and $\xi_{\omega}=0.15 .^{5}$ This will allow us to clarify some aspects reported previously.

When the vector-isovector interaction is absent $\left(\xi_{\rho}=0\right)$, the EoS is harder at high densities (see red curve in Fig. 4, left panel) because the fraction of strangeness is very low. When $\xi_{\rho}$ and $\xi_{\omega}$ are mixed, the larger $\xi_{\rho}$, the lower the onset density of strangeness and, therefore, the larger the fraction of strange quarks. Simultaneously the hadron-quark transition occurs at higher densities and the central densities decreases: the larger $s$-quark contribution softens the quark EoS, and, in order to attain the $2 M_{\odot}$ the contribution of the hadronic star component has to be larger. For example, taking $\xi_{\rho}=0.75$ and $\xi_{\omega}=0.15, \mu_{B}^{H-Q}$ has the highest value when compared with $\mathrm{NJL}(\mathrm{V}+\mathrm{P})$ model for $\xi=$ 0.28 and with $\mathrm{NJL}(\mathrm{V}+\mathrm{P}+\mathrm{VI}+\mathrm{PI})$ model for $\xi=0.22$, while $\rho^{c}$ has the smallest value, as it can be seen in Table VII and in Fig. 4, right panel (for all three cases the maximum gravitational mass is $2 M_{\odot}$ ).

Due to the lack of strangeness in the SU(2) case, the influence of vector-isovector interaction is much smaller when compared with vector-isoscalar interaction. Taking the $2 M_{\odot}$

${ }^{5} \mathrm{By}$ fixing $\xi_{\rho}=0.75$ with $\xi_{\omega}=0$ we have the model $\mathrm{NJL}(\mathrm{VI}+\mathrm{PI})$ for $\xi=0.75$. cases in Table $\mathrm{V}$ it can be seen that the hadron-quark phase transition, and the star properties, are very close for cases with vector-isoscalar interaction $[\mathrm{NJL}(\mathrm{V}+\mathrm{P}+\mathrm{VI}+\mathrm{PI})$ and $\mathrm{NJL}(\mathrm{VI}+\mathrm{PI})$ models]. To have a $2 M_{\odot}$ star with a vectorisovector it is needed a much stronger coupling, however, the hadron-quark phase transition and the star properties are not very different from the other cases (see Table V).

\section{CONCLUSIONS}

We have analyzed the possibility of obtaining hybrid stars with the quark core described within the NJL model with and without strangeness content. Earlier works have shown that only under some conditions a pure quark matter core occurs when quark matter is described within this model. It is, therefore, important to choose adequately the properties of the hadron and the quark phases. In the present work, besides considering the coincidence between the deconfinement phase transition and the partial restoration of chiral symmetry, two new parametrizations of the SU(2) and SU(3) NJL models are proposed with a low vacuum constituent quark mass equal to $313 \mathrm{MeV}$. As shown in [11] a smaller vacuum constituent quark mass favors a hadron-quark phase transition at lower densities and stable stars with a quark core.

We have considered together with the usual scalar and pseudoscalar terms in the NJL model also vector-isoscalar and vector-isovector terms. The vector-isoscalar terms have an important effect on the order of the chiral phase transition and turn the EoS harder [12,14,24]. This, in fact, is also true for the vector-isovector terms, although the EoS does not become so hard and smaller maximum mass configurations are obtained. The inclusion of a vector-isovector term allows larger quark cores, the onset of quark matter at lower densities, smaller hadron-quark mixed phases, and, in the SU(3) version, a larger strangeness content for the same coupling strength. A larger vector-isovector coupling shifts the deconfinement to larger densities and gives rise to a 
smaller quark contribution to the hybrid star properties, mainly if the vector-isoscalar is also considered.

We studied the possibility of getting $2 M_{\odot}$ stars including both vector-isoscalar and vector-isovector terms. It was shown that for the SU(3) NJL $2 M_{\odot}$ configurations always require the presence of a vector-isoscalar term, and that the larger the vector-isovector term the larger the strangeness fraction but the larger the hadron-quark transition density and, therefore, the smaller the quark contribution to the star. It is the $s$-quark with its quite high mass that causes this behavior. In the case of SU(2) NJL, properties of the $2 M_{\odot}$ stars taking different strengths for the vector-isoscalar and isovector terms are almost indistinguishable.

In the present work we have fixed the bag term $B^{*}$ imposing that the deconfinement and the chiral phase transitions coincide. Presently, it is still not clear if both phase transitions coincide, and other scenarios are possible, such as a chiral symmetry restoration before the deconfinement is attained, giving rise to a quarkyonic phase. Imposing different constraints on the $B^{*}$ will have essentially quantitative effects, shifting the onset of quark matter and giving rise to a smaller or larger density jump at the first-order phase transition, but the qualitative features are similar to the ones discussed imposing the coincidence of the chiral and deconfinement transitions.

The main conclusion of the present work is the importance of choosing conveniently the quark model parameters when building a hadron-quark EoS. We have shown that fixing the vacuum quark constituent mass with a value that is one third of the vacuum nucleon mass and, therefore, a baryonic chemical potential at zero density in the quark phase equal to the one in the hadronic phases allows the appearance of a pure quark core in the center of a neutron star. Including a strong enough vector-isoscalar interaction will result in maximum mass configurations with masses above $2 M_{\odot}$. With a vector-isovector interaction alone this is not possible within the SU(3) NJL model, on the other hand, this interaction causes a larger strangeness content and a softening of the quark EoS. However, as in previous studies that have included the strangeness degree of freedom, the strangeness content of these stars is generally very small.

\section{ACKNOWLEDGMENTS}

This work was supported by NewCompStar, COST Action MP1304, and by Fundação para a Ciência e Tecnologia (FCT), Portugal, under the Grant No. SFRH/ BPD/102273/2014 (P. C.), and under the project No. UID/ FIS/04564/2016.

\section{APPENDIX: QUARK PHASE EQUATION OF STATE}

\section{Quark chemical potentials in $\mathbf{S U}(2)$ and $\mathbf{S U}(3)$}

The expressions for the chemical potentials in $\mathrm{SU}(3)$, defined in Eq. (4), are given by: (i) for $\mathrm{NJL}(\mathrm{V}+\mathrm{P}+\mathrm{VI}+\mathrm{PI})$, when $G_{\omega}=G_{\rho}=G_{V}$,

$$
\tilde{\mu}_{i}=\mu_{i}-4 G_{V} \rho_{i} ; \quad i=u, d, s ;
$$

(ii) for $\mathrm{NJL}(\mathrm{V}+\mathrm{P})$, when $G_{\rho}=0$ and $G_{\omega}=G_{V}$,

$$
\begin{aligned}
\tilde{\mu}_{i} & =\mu_{i}-\frac{4}{3} G_{V}\left(\rho_{i}+\rho_{j}+\rho_{k}\right), \\
i & \neq j \neq k \in\{u, d, s\} ;
\end{aligned}
$$

(iii) for $\mathrm{NJL}(\mathrm{VI}+\mathrm{PI})$, when $G_{\omega}=0$ and $G_{\rho}=G_{V}$,

$$
\begin{aligned}
\tilde{\mu}_{i} & =\mu_{i}-\frac{4}{3} G_{V}\left(2 \rho_{i}-\rho_{j}-\rho_{k}\right), \\
i \neq j & \neq k \in\{u, d, s\} .
\end{aligned}
$$

The expressions for the chemical potentials in $\mathrm{SU}(2)$, defined in Eq. (4), are given by:

(i) for $\mathrm{NJL}(\mathrm{V}+\mathrm{P}+\mathrm{VI}+\mathrm{PI})$, when $G_{\omega}=G_{\rho}=G_{V}$,

$$
\tilde{\mu}_{i}=\mu_{i}-4 G_{V} \rho_{i}, \quad i \in\{u, d\}
$$

(ii) for $\mathrm{NJL}(\mathrm{V}+\mathrm{P})$, when $G_{\rho}=0$ and $G_{\omega}=G_{V}$,

$$
\tilde{\mu}_{i}=\mu_{i}-2 G_{V}\left(\rho_{i}+\rho_{j}\right), \quad i \neq j \in\{u, d\} ;
$$

(iii) for $\mathrm{NJL}(\mathrm{VI}+\mathrm{PI})$, when $G_{\omega}=0$ and $G_{\rho}=G_{V}$,

$$
\tilde{\mu}_{i}=\mu_{i}-2 G_{V}\left(\rho_{i}-\rho_{j}\right), \quad i \neq j \in\{u, d\} .
$$

\section{Thermodynamic quantities in $\mathrm{SU}(2)$ and $\mathrm{SU}(3)$}

In $\mathrm{SU}(2)$ as well as in $\mathrm{SU}(3)$, the quark condensate for each flavor is given by:

$$
\sigma_{i}=\left\langle\bar{q}_{i} q_{i}\right\rangle=-2 N_{c} \int \frac{d^{3} p}{(2 \pi)^{3}} \frac{M_{i}}{E_{i}}\left(1-n_{i}-\bar{n}_{i}\right),
$$

where $n_{i}$ and $\bar{n}_{i}$ are the quark and anti-quark occupation numbers:

$$
\begin{aligned}
& n_{i}=\frac{1}{e^{\left(E_{i}-\tilde{\mu}_{i}\right) / T}+1}, \\
& \bar{n}_{i}=\frac{1}{e^{\left(E_{i}+\tilde{\mu}_{i}\right) / T}+1} .
\end{aligned}
$$

The $i$-quark number density, $\rho_{i}=-\left(\partial \Omega / \partial \mu_{i}\right)$ reads

$$
\rho_{i}=2 N_{c} \int \frac{d^{3} p}{(2 \pi)^{3}}\left(n_{i}-\bar{n}_{i}\right) .
$$

The leptonic contribution ( $\beta$-Equilibrium) to the pressure is 
PEREIRA, COSTA, and PROVIDÊNCIA

$$
\begin{aligned}
P^{\beta-\mathrm{eq}}= & P_{\mathrm{NJL}}+2 T \int \frac{d^{3} p}{(2 \pi)^{3}}\left[\ln \left(1+e^{-\left(E_{e}+\mu_{e}\right) / T}\right)\right. \\
& \left.+\ln \left(1+e^{-\left(E_{e}-\mu_{e}\right) / T}\right)\right],
\end{aligned}
$$

being $E_{e}=\sqrt{p^{2}+m_{e}^{2}}$, and to the energy density is

$$
\epsilon^{\beta \text {-eq }}=\epsilon_{\mathrm{NJL}}-2 \int \frac{d^{3} p}{(2 \pi)^{3}} E_{e}\left(n_{e}+\bar{n}_{e}\right),
$$

where $n_{e}$ and $\bar{n}_{e}$ are, respectively,

$$
\begin{gathered}
n_{e}=\frac{1}{e^{\left(E_{e}-\mu_{e}\right) / T}+1} \\
\bar{n}_{e}=\frac{1}{e^{\left(E_{e}+\mu_{e}\right) / T}+1} .
\end{gathered}
$$

The electron density $\left(\rho_{e}=-\left(\partial \Omega_{e} / \partial \mu_{e}\right)\right)$ is given by

$$
\rho_{e}=2 \int \frac{d^{3} p}{(2 \pi)^{3}}\left(n_{e}-\bar{n}_{e}\right) .
$$

In the limit $T=0$ :

$$
\sigma_{i}=\left\langle\bar{q}_{i} q_{i}\right\rangle=-\frac{N_{c}}{\pi^{2}} \int_{\lambda_{F_{i}}}^{\Lambda} \mathrm{d} p p^{2} \frac{M_{i}}{\sqrt{p^{2}+M_{i}^{2}}},
$$

PHYSICAL REVIEW D 94, 094001 (2016)

with the Fermi momentum of the respective quark flavor $i$ given by

$$
\lambda_{F_{i}}=\sqrt{\tilde{\mu}_{i}^{2}-M_{i}^{2}},
$$

and the density given by

$$
\rho_{i}=\frac{N_{c}}{\pi^{2}} \frac{\lambda_{F_{i}}^{3}}{3}
$$

For electrons it comes:

$$
\lambda_{F_{e}}=\sqrt{\tilde{\mu}_{e}^{2}-m_{e}^{2}},
$$

and

$$
\rho_{e}=\frac{\lambda_{F_{i}}^{3}}{3 \pi^{2}}
$$

$$
\begin{aligned}
P_{\mathrm{NJL}}= & -\Omega_{0}-G_{S}\left(\sigma_{u}+\sigma_{d}\right)^{2}+G_{\omega}\left(\rho_{u}+\rho_{d}\right)^{2}+G_{\rho}\left(\rho_{u}-\rho_{d}\right)^{2} \\
& +2 N_{c} \sum_{i=u, d} \int \frac{d^{3} p}{(2 \pi)^{3}}\left[E_{i}+T \ln \left(1+e^{-\left(E_{i}+\tilde{\mu}_{i}\right) / T}\right)+T \ln \left(1+e^{-\left(E_{i}-\tilde{\mu}_{i}\right) / T}\right)\right],
\end{aligned}
$$

and

$$
\begin{aligned}
\epsilon_{\mathrm{NJL}}= & \Omega_{0}+G_{S}\left(\sigma_{u}+\sigma_{d}\right)^{2}-G_{\omega}\left(\rho_{u}+\rho_{d}\right)^{2}-G_{\rho}\left(\rho_{u}-\rho_{d}\right)^{2} \\
& -2 N_{c} \sum_{i=u, d} \int \frac{d^{3} p}{(2 \pi)^{3}}\left[E_{i}\left(1-n_{i}-\bar{n}_{i}\right)+n_{i}\left(\tilde{\mu}_{i}-\mu_{i}\right)+\bar{n}_{i}\left(\mu_{i}-\tilde{\mu}_{i}\right)\right] .
\end{aligned}
$$

In the limit $T=0$ the pressure is given by

$$
P_{\mathrm{NJL}}=-\Omega_{0}-G_{S}\left(\sigma_{u}+\sigma_{d}\right)^{2}+G_{\omega}\left(\rho_{u}+\rho_{d}\right)^{2}+G_{\rho}\left(\rho_{u}-\rho_{d}\right)^{2}+\frac{N_{c}}{\pi^{2}} \sum_{i=u, d} \int_{\lambda_{F_{i}}}^{\Lambda} d p p^{2} E_{i}+\frac{N_{c}}{\pi^{2}} \sum_{i=u, d} \tilde{\mu}_{i} \frac{\lambda_{F_{i}}^{3}}{3},
$$

where the quark density of flavor $f$ is given by Eq. (A18), and energy density is given by

$$
\epsilon_{\mathrm{NJL}}=\Omega_{0}+G_{S}\left(\sigma_{u}+\sigma_{d}\right)^{2}-G_{\omega}\left(\rho_{u}+\rho_{d}\right)^{2}-G_{\rho}\left(\rho_{u}-\rho_{d}\right)^{2}-\frac{N_{c}}{\pi^{2}} \sum_{i=u, d} \int_{\lambda_{F_{i}}}^{\Lambda} d p p^{2} E_{i}+\frac{N_{c}}{\pi^{2}} \sum_{i=u, d}\left(\mu_{i}-\tilde{\mu}_{i}\right) \frac{\lambda_{F_{i}}^{3}}{3} .
$$




\section{b. $\mathrm{SU}(3)$}

The pressure and energy density in $\mathrm{SU}(3)$ are given by:

$$
\begin{aligned}
P_{\mathrm{NJL}}= & -\Omega_{0}-2 G_{S}\left(\sigma_{u}^{2}+\sigma_{d}^{2}+\sigma_{s}^{2}\right)+4 G_{D} \sigma_{u} \sigma_{d} \sigma_{s}+\frac{2}{3} G_{\omega}\left(\rho_{u}+\rho_{d}+\rho_{s}\right)^{2}+G_{\rho}\left(\rho_{u}-\rho_{d}\right)^{2}+\frac{1}{3} G_{\rho}\left(\rho_{u}+\rho_{d}-2 \rho_{s}\right)^{2} \\
& +2 N_{c} \sum_{i=u, d, s} \int \frac{d^{3} p}{(2 \pi)^{3}}\left[E_{i}+T \ln \left(1+e^{-\left(E_{i}+\tilde{\mu}_{i}\right) / T}\right)+T \ln \left(1+e^{-\left(E_{i}-\tilde{\mu}_{i}\right) / T}\right)\right],
\end{aligned}
$$

and

$$
\begin{aligned}
\epsilon_{\mathrm{NJL}}= & \Omega_{0}+2 G_{S}\left(\sigma_{u}^{2}+\sigma_{d}^{2}+\sigma_{s}^{2}\right)-4 G_{D} \sigma_{u} \sigma_{d} \sigma_{s}-\frac{2}{3} G_{\omega}\left(\rho_{u}+\rho_{d}+\rho_{s}\right)^{2}-G_{\rho}\left(\rho_{u}-\rho_{d}\right)^{2}-\frac{1}{3} G_{\rho}\left(\rho_{u}+\rho_{d}-2 \rho_{s}\right)^{2} \\
& -2 N_{c} \sum_{i=u, d, s} \int \frac{d^{3} p}{(2 \pi)^{3}}\left[E_{i}\left(1-n_{i}-\bar{n}_{i}\right)+n_{i}\left(\tilde{\mu}_{i}-\mu_{i}\right)+\bar{n}_{i}\left(\mu_{i}-\tilde{\mu}_{i}\right)\right] .
\end{aligned}
$$

In the limit $T=0$ the pressure becomes,

$$
\begin{aligned}
P_{\mathrm{NJL}}= & -\Omega_{0}-2 G_{S}\left(\sigma_{u}^{2}+\sigma_{d}^{2}+\sigma_{s}^{2}\right)+4 G_{D} \sigma_{u} \sigma_{d} \sigma_{s}+\frac{N_{c}}{\pi^{2}} \sum_{i=u, d, s} \int_{\lambda_{F_{i}}}^{\Lambda} d p p^{2} E_{i}+\frac{N_{c}}{\pi^{2}} \sum_{i=u, d} \tilde{\mu}_{i} \frac{\lambda_{F_{i}}^{3}}{3} \\
& +\frac{2}{3} G_{\omega}\left(\rho_{u}+\rho_{d}+\rho_{s}\right)^{2}+G_{\rho}\left(\rho_{u}-\rho_{d}\right)^{2}+\frac{1}{3} G_{\rho}\left(\rho_{u}+\rho_{d}-2 \rho_{s}\right)^{2},
\end{aligned}
$$

and energy density is,

$$
\begin{aligned}
\epsilon_{\mathrm{NJL}}= & \Omega_{0}+2 G_{S}\left(\sigma_{u}^{2}+\sigma_{d}^{2}+\sigma_{s}^{2}\right)-4 G_{D} \sigma_{u} \sigma_{d} \sigma_{s}-\frac{N_{c}}{\pi^{2}} \sum_{i=u, d, s} \int_{\lambda_{F_{i}}}^{\Lambda} d p p^{2} E_{i}+\frac{N_{c}}{\pi^{2}} \sum_{i=u, d}\left(\mu_{i}-\tilde{\mu}_{i}\right) \frac{\lambda_{F_{i}}^{3}}{3} \\
& -\frac{2}{3} G_{\omega}\left(\rho_{u}+\rho_{d}+\rho_{s}\right)^{2}-G_{\rho}\left(\rho_{u}-\rho_{d}\right)^{2}-\frac{1}{3} G_{\rho}\left(\rho_{u}+\rho_{d}-2 \rho_{s}\right)^{2} .
\end{aligned}
$$

[1] J. Antoniadis et al., A massive pulsar in a compact relativistic binary, Science 340, 6131 (2013).

[2] P. Demorest, T. Pennucci, S. Ransom, M. Roberts, and J. Hessels, Shapiro delay measurement of a two solar mass neutron star, Nature (London) 467, 1081 (2010).

[3] E. Fonseca et al., The NANOGrav nine-year data set: Mass and geometric measurements of binary millisecond pulsars, arXiv:1603.00545.

[4] N. K. Glendenning, Compact Stars (Springer-Verlag, New York, 2000).

[5] A. Chodos, R. L. Jaffe, K. Johnson, and C. B. Thorn, Baryon structure in the bag theory, Phys. Rev. D 10, 2599 (1974).

[6] I. Bombaci, I. Parenti, and I. Vidana, Quark deconfinement and implications for the radius and the limiting mass of compact stars, Astrophys. J. 614, 314 (2004).

[7] K. Schertler, S. Leupold, and J. Schaffner-Bielich, Neutron stars and quark phases in the NJL model, Phys. Rev. C 60, 025801 (1999).

[8] M. Baldo, M. Buballa, F. Burgio, F. Neumann, M. Oertel, and H. J. Schulze, Neutron stars and the transition to color superconducting quark matter, Phys. Lett. B 562, 153 (2003).
[9] D. P. Menezes and C. Providencia, Warm stellar matter with deconfinement: Application to compact stars, Phys. Rev. C 68, 035804 (2003).

[10] I. Shovkovy, M. Hanauske, and M. Huang, Nonstrange hybrid compact stars with color superconducting matter, Phys. Rev. D 67, 103004 (2003).

[11] M. Buballa, F. Neumann, M. Oertel, and I. Shovkovy, Quark mass effects on the stability of hybrid stars, Phys. Lett. B 595, 36 (2004).

[12] G. Pagliara and J. Schaffner-Bielich, Stability of CFL cores in hybrid stars, Phys. Rev. D 77, 063004 (2008).

[13] M. Buballa and M. Oertel, Strange quark matter with dynamically generated quark masses, Phys. Lett. B 457, 261 (1999).

[14] L. Bonanno and A. Sedrakian, Composition and stability of hybrid stars with hyperons and quark color-superconductivity, Astron. Astrophys. 539, A16 (2012).

[15] D. Logoteta, C. Providência, and I. Vidaña, Formation of hybrid stars from metastable hadronic stars, Phys. Rev. C 88, 055802 (2013).

[16] D. Blaschke, J. Schaffner-Bielich, and Hans-Josef Schulze, Topical issue on exotic matter in neutron stars, Eur. Phys. J. A 52, 71 (2016). 
PEREIRA, COSTA, and PROVIDÊNCIA

[17] G. F. Burgio and D. Zappalà, Hybrid star structure with the field correlator method, Eur. Phys. J. A 52, 60 (2016).

[18] E. S. Fraga, A. Kurkela, and A. Vuorinen, Neutron star structure from QCD, Eur. Phys. J. A 52, 49 (2016).

[19] A. Drago, M. Fiolhais, and U. Tambini, Quark matter in the chiral color dielectric model, Nucl. Phys. A588, 801 (1995).

[20] D. Logoteta, I. Bombaci, C. Providencia, and I. Vidana, A chiral model approach to quark matter nucleation in neutron stars, Phys. Rev. D 85, 023003 (2012).

[21] D. Alvarez-Castillo, A. Ayriyan, S. Benic, D. Blaschke, H. Grigorian, and S. Typel, New class of hybrid EoS and Bayesian M-R data analysis, Eur. Phys. J. A 52, 69 (2016).

[22] S. Lawley, W. Bentz, and A. W. Thomas, Nucleons, nuclear matter and quark matter: A unified NJL approach, J. Phys. G 32, 667 (2006).

[23] H. Pais, D. P. Menezes, and C. Providência, Neutron stars: From the inner crust to the core with the (extended) Nambu-Jona-Lasinio model, Phys. Rev. C 93, 065805 (2016).

[24] M. Hanauske, L. M. Satarov, I. N. Mishustin, H. Stoecker, and W. Greiner, Strange quark stars within the Nambu-JonaLasinio model, Phys. Rev. D 64, 043005 (2001).

[25] T. Klahn, D. Blaschke, F. Sandin, C. Fuchs, A. Faessler, H. Grigorian, G. Ropke, and J. Trumper, Modern compact star observations and the quark matter equation of state, Phys. Lett. B 654, 170 (2007).

[26] C. H. Lenzi and G. Lugones, Hybrid stars in the light of the massive pulsar PSR J1614 - 2230, Astrophys. J. 759, 57 (2012).

[27] K. Masuda, T. Hatsuda, and T. Takatsuka, Hadron-quark crossover and massive hybrid stars, Prog. Theor. Exp. Phys. (2013) 073D01.

[28] T. Klähn, R. Łastowiecki, and D. B. Blaschke, Implications of the measurement of pulsars with two solar masses for quark matter in compact stars and heavy-ion collisions: A Nambu-Jona-Lasinio model case study, Phys. Rev. D 88, 085001 (2013).

[29] D. P. Menezes, M. B. Pinto, L. B. Castro, P. Costa, and C. Providência, Repulsive vector interaction in three flavor magnetized quark and stellar matter, Phys. Rev. C 89, 055207 (2014).

[30] T. Klahn and T. Fischer, Vector interaction enhanced bag model for astrophysical applications, Astrophys. J. 810, 134 (2015).

[31] E. J. Ferrer, V. de la Incera, and L. Paulucci, Gluon effects on the equation of state of color superconducting strange stars, Phys. Rev. D 92, 043010 (2015).

[32] K. Fukushima, Phase diagrams in the three-flavor NambuJona-Lasinio model with the Polyakov loop, Phys. Rev. D 77, 114028 (2008); 78, 039902(E) (2008).

[33] S. Klimt, M. F. M. Lutz, U. Vogl, and W. Weise, Generalized SU(3) Nambu-Jona-Lasinio model. Part. 1. Mesonic modes, Nucl. Phys. A516, 429 (1990).

[34] M. F. M. Lutz, S. Klimt, and W. Weise, Meson properties at finite temperature and baryon density, Nucl. Phys. A542, 521 (1992).
PHYSICAL REVIEW D 94, 094001 (2016)

[35] N. M. Bratovic, T. Hatsuda, and W. Weise, Role of vector interaction and axial anomaly in the PNJL modeling of the QCD phase diagram, Phys. Lett. B 719, 131 (2013).

[36] O. Lourenco, M. Dutra, T. Frederico, A. Delfino, and M. Malheiro, Vector interaction strength in Polyakov-NambuJona-Lasinio models from hadron-quark phase diagrams, Phys. Rev. D 85, 097504 (2012).

[37] P. Rehberg, S. P. Klevansky, and J. Hufner, Hadronization in the SU(3) Nambu-Jona-Lasinio model, Phys. Rev. C 53, 410 (1996).

[38] C. J. Horowitz and J. Piekarewicz, Neutron Star Structure and the Neutron Radius of Pb-208, Phys. Rev. Lett. 86, 5647 (2001).

[39] M. Fortin, C. Providencia, A. R. Raduta, F. Gulminelli, J. L. Zdunik, P. Haensel, and M. Bejger, Neutron star radii and crusts: Uncertainties and unified equations of state, Phys. Rev. C 94, 035804 (2016).

[40] P. Costa, M. C. Ruivo, C. A. de Sousa, and Y. L. Kalinovsky, Analysis of the $\mathrm{U}(\mathrm{A})(1)$ symmetry-breaking and restoration effects on scalar-pseudoscalar spectrum, Phys. Rev. D 71, 116002 (2005).

[41] M. Buballa, NJL model analysis of quark matter at large density, Phys. Rep. 407, 205 (2005).

[42] J. Moreira, B. Hiller, A. A. Osipov, and A. H. Blin, Thermodynamic potential with correct asymptotics for PNJL model, Int. J. Mod. Phys. A 27, 1250060 (2012).

[43] K. A. Olive et al. (Particle Data Group Collaboration), Review of particle physics, Chin. Phys. C 38, 090001 (2014).

[44] P. Costa, M. C. Ruivo, C. A. de Sousa, and H. Hansen, Phase diagram and critical properties within an effective model of QCD: the Nambu-Jona-Lasinio model coupled to the Polyakov loop, Symmetry 2, 1338 (2010).

[45] J. R. Oppenheimer and G. M. Volkoff, On massive neutron cores, Phys. Rev. 55, 374 (1939).

[46] R. C. Tolman, Static solutions of Einstein's field equations for spheres of fluid, Phys. Rev. 55, 364 (1939).

[47] G. Baym, C. Pethick, and P. Sutherland, The ground state of matter at high densities: Equation of state and stellar models, Astrophys. J. 170, 299 (1971).

[48] F. Grill, H. Pais, C. Providência, I. Vidaña, and S. S. Avancini, Equation of state and thickness of the inner crust of neutron stars, Phys. Rev. C 90, 045803 (2014).

[49] F. Ozel, D. Psaltis, T. Guver, G. Baym, C. Heinke, and S. Guillot, The dense matter equation of state from neutron star radius and mass measurements, Astrophys. J. 820, 28 (2016).

[50] A. W. Steiner, J. M. Lattimer, and E. F. Brown, Neutron star radii, universal relations, and the role of prior distributions, Eur. Phys. J. A 52, 18 (2016).

[51] V. F. Suleimanov, J. Poutanen, D. Klochkov, and K. Werner, Measuring the basic parameters of neutron stars using model atmospheres, Eur. Phys. J. A 52, 20 (2016).

[52] W. C. Chen and J. Piekarewicz, Compactness of Neutron Stars, Phys. Rev. Lett. 115, 161101 (2015).

[53] P. Haensel, M. Bejger, M. Fortin, and L. Zudnik, Rotating neutron stars with exotic cores: masses, radii, stability, Eur. Phys. J. A 52, 59 (2016). 